\title{
Impact of Classical Control Electronics on Qubit Fidelity
}

\author{
J.P.G. van Dijk,,${ }^{1,2,}{ }^{*}$ E. Kawakami, ${ }^{3}$ R.N. Schouten,,${ }^{1,2}$ M. Veldhorst,,${ }^{1,2}$ L.M.K. Vandersypen,,${ }^{1,2,4}$ \\ M. Babaie, ${ }^{1}$ E. Charbon, ${ }^{1,2,4,5}$ and F. Sebastiano ${ }^{1}$ \\ ${ }^{1}$ QuTech, Delft University of Technology, P.O. Box 5046, 2600 GA Delft, Netherlands \\ ${ }^{2}$ Kavli Institute of Nanoscience, P.O. Box 5046, 2600 GA Delft, Netherlands \\ ${ }^{3}$ Okinawa Institute of Science and Technology, Okinawa 904-0412, Japan \\ ${ }^{4}$ Intel Corporation, $2501 \mathrm{NW} 229$ th Ave, Hillsboro Oregon 97124, USA \\ ${ }^{5}$ École Polytechnique Fédérale de Lausanne, Case postale 526, CH-2002 Neuchâtel, Switzerland
}

(Received 17 March 2018; revised manuscript received 30 May 2019; published 24 October 2019)

\begin{abstract}
Quantum processors rely on classical electronic controllers to manipulate and read out the state of quantum bits (qubits). As the performance of the quantum processor improves, nonidealities in the classical controller can become the performance bottleneck for the whole quantum computer. To prevent such limitation, this paper presents a systematic study of the impact of the classical electrical signals on the qubit fidelity. All operations, i.e., single-qubit rotations, two-qubit gates, and readout, are considered, in the presence of errors in the control electronics, such as static, dynamic, systematic, and random errors. Although the presented study could be extended to any qubit technology, it currently focuses on singleelectron spin qubits, because of several advantages, such as purely electrical control and long coherence times, and for their potential for large-scale integration. As a result of this study, detailed electrical specifications for the classical control electronics for a given qubit fidelity can be derived. We also discuss how qubit fidelity is affected by the limited performance of the general-purpose room-temperature equipment typically employed to control the few qubits available today. Ultimately, we show that tailor-made electronic controllers can achieve significantly lower power, cost, and size, as required to support the scaling up of quantum computers.
\end{abstract}

DOI: 10.1103/PhysRevApplied.12.044054

\section{INTRODUCTION}

Quantum computers have the potential to solve problems that are intractable even for the most powerful supercomputers [1]. These problems include the factorization of prime numbers using Shor's algorithm [2], the efficient search in large data sets using Grover's algorithm [3], and the simulation of quantum systems as initially proposed by Feynman [4]. A quantum computer operates by processing the information stored in quantum bits (qubits), which are organized in a quantum processor. Performing operations on the qubits requires a classical electronic controller for manipulating the qubits and reading out their quantum state [5]. In order not to degrade qubit performance, the classical controller must provide high-accuracy low-noise control signals and the readout

\footnotetext{
*j.p.g.vandijk@tudelft.nl
}

Published by the American Physical Society under the terms of the Creative Commons Attribution 4.0 International license. Further distribution of this work must maintain attribution to the author(s) and the published article's title, journal citation, and DOI. must be very sensitive and quiet to detect the weak signals from the quantum processor without altering the qubit states.

Since state-of-the-art quantum processors comprise only a few qubits ( $<20$ qubits [6-8]), the classical controller is currently typically implemented by general-purpose instruments operating at room temperature or tailor-made room-temperature controllers $[9,10]$. For a recent review of state-of-the-art electronic interface for quantum processors, see Ref. [11]. The use of these high-performance instruments results in the fidelity of the quantum operations being limited by the quantum processor [12]. However, as the performance of the quantum processor improves, the classical controller can become performance limiting. Consequently, it is crucial to understand how the controller impacts the performance of the whole quantum computer in order to properly codesign the controller and the quantum processor and to identify potential performance bottlenecks.

Moreover, the simplest nontrivial algorithms, such as quantum-chemistry problems, require more than 100 logical qubits [13]. This translates into the need for thousands or millions of physical qubits, if the redundancy added by 
quantum-error correction schemes, such as surface codes [14], is taken into account. For such large-scale quantum processors, implementation of the classical controller with general-purpose instruments would be impractical and would offer limited scalability due to its size and cost. A more practical and power-efficient approach would be to use tailor-made electronics that can be optimized for this specific application in terms of power consumption, form factor, and cost [5,11,15-23]. However, defining the specifications to design such electronics requires a comprehensive analysis of the impact of the electronics performance on the quantum computer.

Furthermore, solid-state qubits need to be cooled to deep cryogenic temperatures. When operating the control electronics at cryogenic temperatures to relax the wiring requirements between the cryogenic quantum processor and its controller, as proposed in Refs. [5,15-20], the need for accurate specifications is even more severe. The power dissipation of such a cryogenic controller is limited by the cooling power of the cryogenic refrigerator. For existing fridges, this is only about $1 \mathrm{~W}$ at $4 \mathrm{~K}$ and $<1 \mathrm{~mW}$ below $100 \mathrm{mK}$ [24]. Although this could improve in the future by, e.g., adopting custom-made refrigerators [25], the power consumption of the controller is also expected to increase to serve an increasing number of qubits. To meet these cooling constraints, the power dissipated by the electronics must be minimized by optimally allocating the available power across the various components of the classical controller. However, carrying out such optimization also demands a clear understanding of the impact of each component on the quantum-computer performance.

Analysis of the impact of the controller on the quantum computer's performance has been undertaken previously, but only for specific aspects of the control signals, i.e., the effect of microwave phase noise [26,27], or for theoretically treating the effects of noise on qubit operations in a filter function framework [28,29], and control sweet spots [30-32] and noise effects in general [33-35]. The work presented here aims to provide a comprehensive analysis of the effect of nonideal circuit blocks in the classical controller on the qubit fidelity for all possible operations, i.e., single-qubit gates, two-qubit gates, and readout. Initialization is assumed to be performed by relaxation or by readout and will not be separately discussed. This includes the effect of signal inaccuracies in the frequency, voltage, and time domain, and covers static, dynamic, systematic, and random errors. Only with a full set of specifications can potential bottlenecks be identified and tailor-made electronics designed. Besides providing a general method for deriving the electronics specifications, the specifications resulting from a case study targeting a $99.9 \%$ average gate fidelity are mapped onto existing room-temperature integrated circuits (IC) to assess the feasibility of a practical controller.
Although the proposed approach can be easily extended to any quantum technology, such as NMR [36-38], ion traps [8,39], superconducting qubits [7,40,41], or nitrogenvacancy $(\mathrm{N}-V)$ centers in diamond [42], we focus on the specific case of single-electron spin qubits. This qubit technology offers promising prospects for large-scale quantum computing, due to the long coherence times [12,43], the fully electrical control $[44,45]$, and the potential integration of the quantum processor with a classical controller on a single chip fabricated using standard microelectronic technologies [46]. In addition, the results obtained for the single-qubit gates can be generalized to any qubit system where single-qubit rotations are performed by applying a signal with a frequency matching the energy-level spacing between the $|0\rangle$ and $|1\rangle$ states, e.g., for NMR [36-38], ion traps [8,39], N- $V$ centers in diamond [42], and superconducting qubits $[7,40,41]$. Similarly, the results obtained for the two-qubit gates can be generalized to any qubit system that exploits the exchange gate.

The paper is organized as follows: Sec. II describes the generalized spin-qubit quantum computer analyzed in this paper; Sec. III introduces the method for deriving the fidelity for the various operations; and in Secs. IV, V, and VI the electrical specifications required for single-qubit operations, two-qubit operations, and qubit readout are derived, respectively. A discussion regarding the feasibility of these specifications follows in Sec. VII. Conclusions are drawn in Sec. VIII.

\section{A SYSTEM-LEVEL VIEW OF A QUANTUM COMPUTER}

\section{A. The quantum processor}

A single-electron spin qubit encodes the quantum state in the spin state of a single electron. A generic model of a quantum processor based on single-electron spin qubits is shown in Fig. 1(a), which captures all of the properties relevant for the interaction with the controller. Moreover, the figure illustrates a linear array of quantum dots but this can be extended to more complex geometries such as a 2D grid of quantum dots, as shown in Refs. [46-48].

Quantum dots are formed using a set of gate electrodes that locally deplete a two-dimensional electron gas (2DEG) on a semiconductor chip (e.g., a GaAs/(Al,Ga)As heterojunction, a $\mathrm{Si} / \mathrm{SiGe}$ heterojunction, or a $\mathrm{Si}-$ metaloxide semiconductor (MOS) structure $[49,50]$ ). Due to the small size of the quantum dot, the charge states become discrete with an energy-level spacing related to the dot charging energy, thereby setting the required increase of the dot potential to add an electron to the dot. The dot potential, and thereby the number of electrons in the dot, is controlled by the plunger gate that capacitively couples to the quantum dot. Without loss of generality for the analysis of the electrical control signals, the following analysis assumes the availability of additional tunnel-barrier gates 
(a)

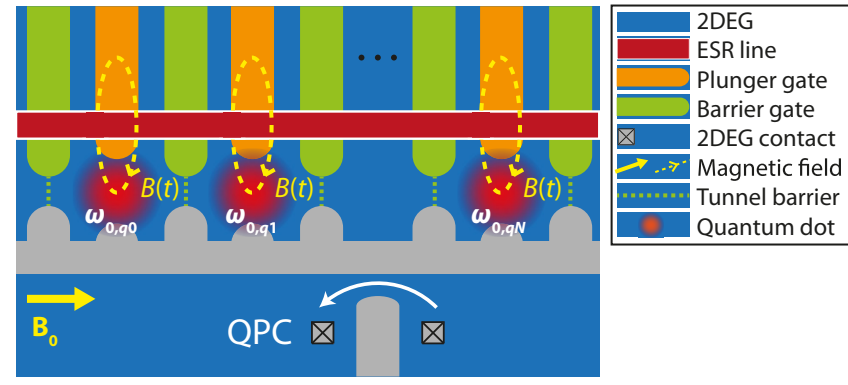

(b)

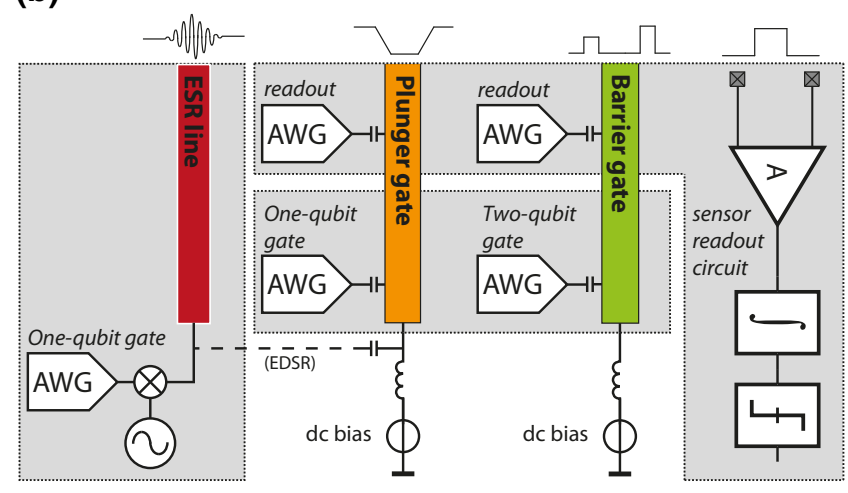

FIG. 1. (a) A generic model of a spin-qubit quantum processor comprising qubits encoded in the spin of electrons trapped in quantum dots and a charge sensor (e.g., a quantum point contact, QPC). The blue background indicates the two-dimensional electron gas (2DEG) where quantum dots, shown in red, are formed locally. Individual control over the dot potential and the tunnel barriers is assumed, using plunger gates (orange) and barrier gates (green), respectively. Furthermore, each qubit can have a unique Larmor frequency $\left(\omega_{0, q i}\right)$. (b) The classical control electronics required for each line type (electron-spin-resonance (ESR) line, plunger gate, and barrier gate) of the quantum processor. The electronic components in the figure are placeholders for the respective functionalities and are grouped by operation, i.e., single-qubit operation, two-qubit operation, and readout. Thus, they do not necessarily correspond to a physical implementation. Arbitrary-waveform generators (AWG) are shown for the envelope and pulse generation.

that form tunnel barriers between neighboring dots by controlling the width of the depletion layer, thus allowing tunneling of electrons from and to the quantum dot in a tunable way. Early integration schemes involved nonoverlapping gates [as shown in Fig. 1(a)] [44,51,52], while, in order to create better tunability and control, architectures now often include overlapping gates [45,53-55].

An external static magnetic field $\mathbf{B}_{0}$ induces an energy difference between electrons with spin up and spin down, with Zeeman energy $E_{z}$. Because of the static magnetic field, the electron rotates around the $Z$ axis in the Bloch sphere with Larmor frequency $\omega_{0}=\gamma_{e}\left|\mathbf{B}_{0}\right|$, where $\gamma_{e}$ is the gyromagnetic ratio of the electron $\left(\gamma_{e} \approx 28 \mathrm{GHz} / \mathrm{T}\right.$ in silicon). As indicated in Fig. 1(a), each qubit can have a different Larmor frequency, which can be useful for two-qubit operations or for multiplexing single-qubit operations [45,55-57].

Single-qubit operations (Sec. IV) require the application of a varying magnetic field perpendicular to $\mathbf{B}_{0}$ and oscillating at the Larmor frequency. In the case of electron spin resonance (ESR), such a field is generated by a varying current in a nearby ESR line $[45,55,58]$. Alternatively, the same effect can be obtained, e.g., by applying a varying electric field to the electron in a spatial magnetic field gradient, as is the case for electric-dipole spin resonance (EDSR) $[44,52,59,60]$. In that case, the electric field variations are generated by a voltage on a nearby gate, e.g., through the plunger gate, without requiring an ESR line. Although Fig. 1(a) shows an ESR line, the results of the analysis below are applicable to both ESR- or EDSRbased operations, as explained in the following section. Two-qubit operations (Sec. V) and qubit readout (Sec. VI) can be performed by pulsing the barrier and plunger gates.

The effect of cross talk between different gates or the ESR line is considered negligible or compensated for in the classical controller and is not further discussed here, since it can be treated as a purely classical electrical effect.

Qubit readout relies on a spin-to-charge conversion, followed by the detection of the eventual electron movement [49], using either a gate-dispersive readout [61] or an additional charge sensor. The latter is assumed in this paper, as gate-based readout is still under development [62]. For such a charge sensor, different sensing techniques can be used, e.g., a QPC [63-65] or a single-electron transistor (SET) [66]. As an example, Fig. 1(a) shows a QPC in close proximity to the quantum dots.

\section{B. The classical electronic controller}

The classical controller is responsible for generating the required electrical signals to bias and control each gate and, in case, the ESR line, and for reading the state of the charge sensor. The required electronics are schematically summarized in Fig. 1(b).

When no operation is performed, each quantum dot must contain a single electron at the same dot potential and the tunnel barriers must be tuned to ensure a negligible coupling between neighboring dots (Sec. VB). Such conditions are ensured by the use of bias-voltage generators, as shown in Fig. 1(b).

The oscillating magnetic field $B(t)$ required for singlequbit operations can be generated by an oscillating current $I(t)$, following the relation $B(t)=\alpha_{I} I(t)$ (in the case of ESR), or by an oscillating voltage $V(t)$, resulting in $B(t)=$ $\alpha_{V} V(t)$ (in the case of EDSR). The conversion factors $\alpha_{I}$ and $\alpha_{V}$ depend on many factors, such as the exact geometry of the structures, and can be determined experimentally. Due to this abstraction, the results shown in Sec. IV are valid both in the case of ESR and EDSR. 
The required microwave current or voltage is generated by modulating a carrier from a local oscillator (LO) with an envelope produced by an AWG. Although different hardware implementations are possible, this allows us, without loss of generality, to split the carrier specifications, i.e., the local oscillator specifications, from the envelope specifications, i.e., the AWG specifications. In the case in which each qubit has a unique Larmor frequency, a single control line can be used to control multiple qubits independently via frequency-division multiple access (FDMA), i.e., frequency multiplexing, thus simplifying the wiring (Sec. IV B).

The voltage pulses required for the two-qubit gates and readout are generated by AWGs. Distinct AWGs are assumed for two-qubit gates and readout since the specifications for such operations can be different.

Besides the presented control electronics, additional hardware is required to process the signal from the charge sensor. The required hardware depends on the readout method employed, e.g., a direct measurement [64] or radiofrequency (rf) reflectometry [61,63]. As an example, a direct readout, requiring a readout amplifier, is shown in Fig. 1(b).

\section{METHODS}

The evolution of the qubit state is evaluated by computing the system Hamiltonian $(H)$, which is a function of the electrical signals applied by the classical controller. For static control signals, the Hamiltonian is time independent and the unitary operation describing the evolution after a time $T$ is trivially $U=e^{-i H T}(\hbar=1)$.

For dynamic signals, such as for complex signal envelopes, the operation described by the time-varying Hamiltonian $H(t)$ is approximated by the product of timeindependent components, leading to

$$
U \approx \prod_{n=N}^{0} e^{-i H(n \Delta t) \Delta t},
$$

where $\Delta t$ is the time step, which must be chosen to be small enough for the required accuracy of the approximation.

As a benchmark to evaluate how close $U$ is to the operation from an ideal controller $U_{\text {ideal }}$, the process fidelity is computed as follows $[67,68]$ :

$$
F=\frac{1}{n^{2}}\left|\operatorname{Tr}\left[U_{\text {ideal }}^{\dagger} U\right]\right|^{2},
$$

for the $n$-dimensional complex Hilbert space ( $n=2$ for the single-qubit gate and $n=4$ for the two-qubit gate).

In case of random dynamic errors, the ensemble average over all realizations is evaluated, following Refs. [28] and [29]. When treating random noise, the noise spectrum is relevant, as the operation can be affected differently by noise at different frequencies. The method presented in Refs. [28] and [29] is used to evaluate the expected process fidelity and is outlined in the Supplemental Material [69].

Detailed derivations of the analytical results presented in this paper are collected in the Supplemental Material [69]. The analytical results presented in the main text are often the result of a series expansion of the fidelity truncated at the second order, as clearly stated in the Supplemental Material [69]. Since this study is focused on high qubit fidelity (> 90\%), higher-order processes can be neglected. All presented results are verified by numerical simulations of the Hamiltonian.

\section{SIGNAL SPECIFICATIONS FOR SINGLE-QUBIT OPERATIONS}

\section{A. Fidelity of a single-qubit operation}

As explained in Sec. II, the qubit rotates around the $Z$ axis due to the applied external magnetic field. Using an LO tuned to a frequency equal to the qubit's Larmor frequency, the qubit phase can be tracked and the qubit appears to be stationary in the reference frame of the LO. In this rotating frame, $Z$ rotations by an angle $\theta_{Z}$ can easily be obtained by instantaneously updating the LO's phase in software by an angle $\theta_{Z}[37,38]$. For such a softwaredefined $Z$ rotation, only the accuracy of the phase update of the LO matters, which is limited by the finite resolution in the phase setting. A phase error $\Delta \phi=\Delta \theta_{Z}$ reduces the fidelity of the $Z$ rotation as follows:

$$
F_{Z_{\phi}}=1-\frac{1}{4} \Delta \phi^{2} .
$$

In the remainder of this section, we will focus on rotations around the $X$ or $Y$ axis that are obtained by applying a magnetic field $B(t)$ oscillating at the qubit Larmor frequency $\omega_{0}$ and with a specific phase, which is generated by applying either a microwave current or a microwave voltage, as explained in Sec. II. The Hamiltonian describing a single electron under microwave excitation in the laboratory frame is given by the following $(\hbar=1)$ :

$$
H_{\mathrm{lab}}=-\omega_{0} \frac{\sigma_{z}}{2}+\gamma_{e} B(t) \frac{\sigma_{x}}{2},
$$

where, here and in the following, $\sigma_{x}, \sigma_{y}$, and $\sigma_{z}$ are the Pauli matrices. The microwave magnetic field can be described as $B(t)=2 / \gamma_{e} \omega_{R}(t) \cos \left(\omega_{\mathrm{mw}} t+\phi\right)$. A constant amplitude $\left[\omega_{R}(t)=\omega_{R}\right]$, i.e., a rectangular envelope, is considered, unless stated otherwise. In the case of a rectangular envelope, $\omega_{R}$ is the Rabi frequency, i.e., the rotation speed for the single-qubit gate. Note that for more complex envelopes, the resulting specifications for the control electronics can differ, as the sensitivity to certain control parameters can be reduced when employing quantum optimum control, such as GRAPE [70]. 
For single-qubit rotations, the rotation axis is affected by the matching of the microwave frequency $\left(\omega_{\mathrm{mw}}\right)$ to the Larmor frequency $\left(\omega_{0}\right)$ and by the phase of the microwave signal $(\phi)$, i.e., the carrier signal. The rotation angle $(\theta=$ $\left.\omega_{R} T\right)$, on the other hand, is determined by the amplitude of the signal $\left(\omega_{R}\right)$ and the duration for which the microwave signal is applied $(T)$, i.e., the signal envelope.

Table I summarizes the effect of noise and inaccuracy on the fidelity of single-qubit operations. Inaccuracies in the control signal are generally caused by instrument finite resolution and drift [71]. In the case of envelope inaccuracies, the microwave amplitude $\left(\propto \omega_{R}\right)$ and duration $(T)$ of the signal together determine the rotation angle $\left(\theta=\omega_{R} T\right)$. Hence, any error in either one leads to an under or over rotation, thereby reducing the fidelity. In the case of frequency inaccuracies, a better fidelity is achieved for larger Rabi frequencies, i.e., a larger microwave amplitude and a shorter pulse duration. However, a larger Rabi frequency ultimately requires a sufficiently larger Larmor frequency [72] and it is harder to reach the same phase accuracy $\Delta \phi$ at higher $\mathrm{LO}$ frequencies.

Next, dynamic changes in the control signal can further limit the fidelity. In the event that such a change occurs on a time scale larger than the operation time, it can be considered a random static error. For a static but random error $\Delta$ for which $F=1-c \Delta^{2}$, the expected fidelity simply follows as $F=1-c \sigma^{2}$, if $\Delta$ follows a Gaussian distribution with standard deviation $\sigma$ and zero mean (see the Supplemental Material [69]). Hence the equations for the inaccuracy as given in Table I apply.

In order to treat random noise with spectral content at frequencies higher than the operation rate, the method presented in Refs. [28] and [29] is adopted to compute the expected operation fidelity as a function of the noise spectrum. The results for dephasing noise are reproduced from Refs. [26,28,29] and are repeated here for completeness, with additional analysis for different rotation angles. The fidelity due to the various noise sources is summarized in Table I, where generally $|H(\omega)|^{2}$ is the intrinsic qubit-filter function, implying that the qubit has a different sensitivity to noise at different frequencies. The amplitude response of the intrinsic qubit-filtering functions for frequency noise and amplitude noise are shown in Fig. 2, with analytical formulas provided in the Supplemental Material [69]. These responses have a low-pass filter (LPF) characteristic and their properties, the dc gain and the effective noise bandwidth (ENBW), are summarized in Table I and highlighted in the plots as the brick-wall approximation of the filter [73].

Note that for frequency noise, the ENBW is proportional to the Rabi frequency, indicating that for faster operations, noise in a wider band affects the qubit. However, the lower limit of integration $\left(\omega_{\min }\right)$ is inversely proportional to the execution time of the quantum algorithm [74] and is therefore also related to the operation time. In the

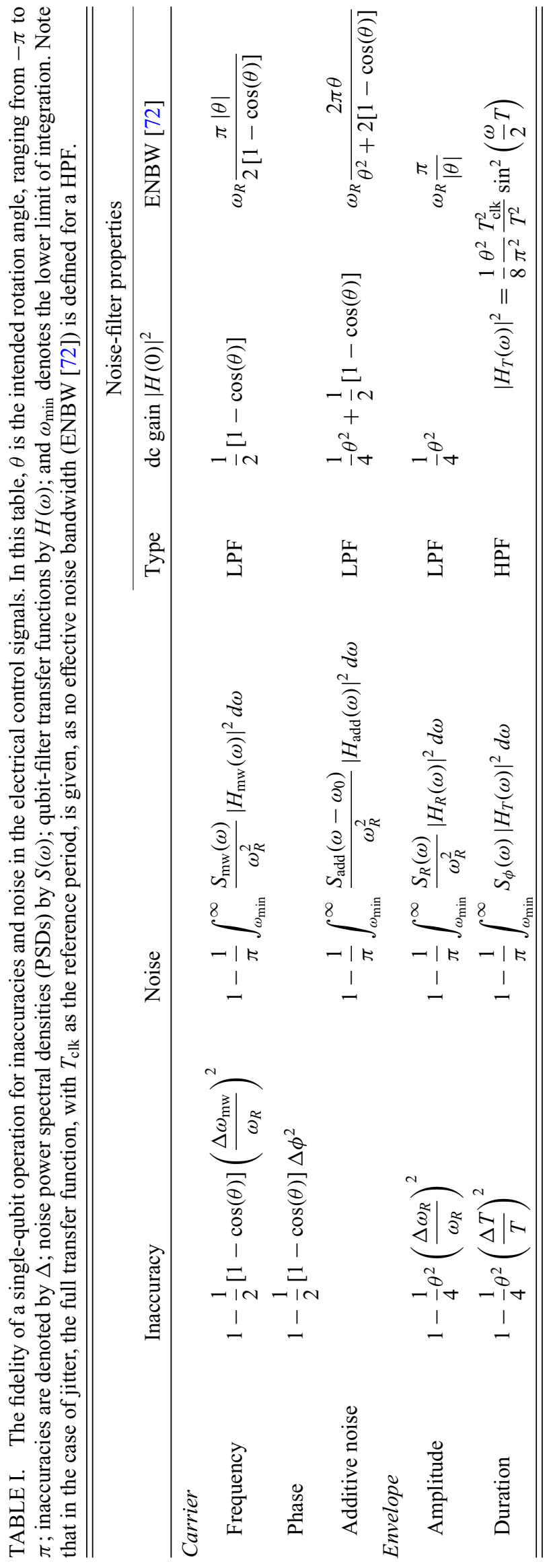



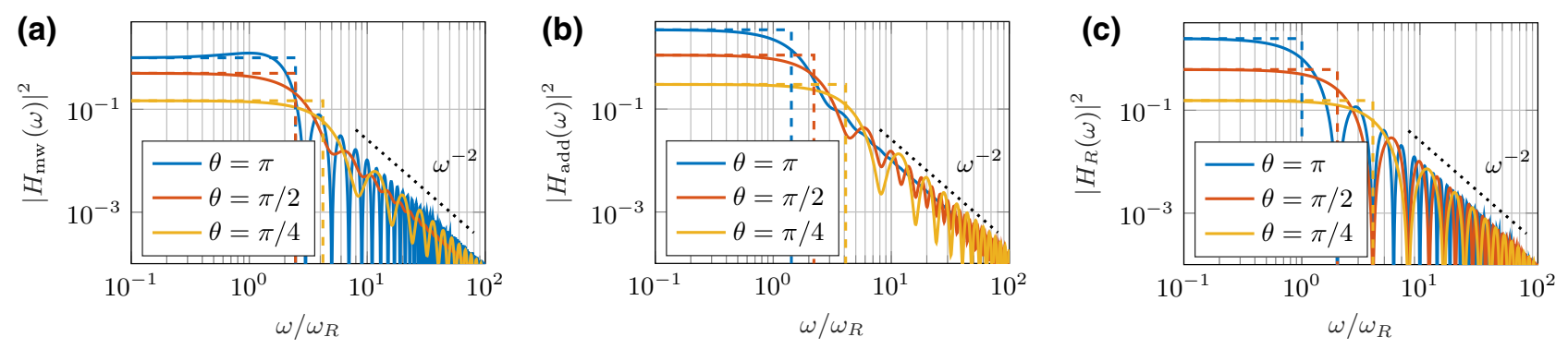

FIG. 2. The amplitude response of the intrinsic qubit filter for (a) frequency noise, (b) wide-band additive noise, and (c) amplitude noise, for various rotation angles $\theta$. The brick-wall approximations are shown with dashed lines.

case of white noise, a good approximation is obtained with $\omega_{\min }=0$. Due to the factor $1 / \omega_{R}^{2}$, it is advantageous to use the highest possible Rabi frequency. In the presence of flicker noise, the same conclusion holds, as then a higher $\omega_{\min }$ is desirable. Finally, the same discussion holds in the case of amplitude noise. However, a higher tolerance for amplitude noise at larger Rabi frequencies, i.e., larger amplitudes, simply means that the required signal-to-noise ratio $\left(\omega_{R}^{2} / \sigma_{\omega_{R}}^{2}\right)$ in the qubit's band of sensitivity is fixed.

The microwave-frequency noise $\left[S_{\omega}(\Delta \omega)\right]$, as discussed before, is set by the phase noise $\left[S_{\phi}(\Delta \omega)\right]$ of the LO generator and they can be related by $S_{\omega}(\Delta \omega)=\Delta \omega^{2} S_{\phi}(\Delta \omega)$ at a frequency $\Delta \omega$ from the carrier $\omega_{\mathrm{mw}}$ [26]. While the effect of phase noise has already been extensively studied in Ref. [26], a more realistic oscillator noise model, including both phase noise and wide-band additive noise, is adopted in this work. Derivation of the intrinsic qubitfiltering function for each noise contribution leads to an improved estimation of the fidelity that deviates from Ref. [26], as elaborated in the following.

Consider, as an example, the typically reported plot for the phase noise of a phase-locked-loop- (PLL) based frequency generator, as shown in Fig. 3 (cf. Ref. [75]). Appended to this figure is a plot of the resulting frequencynoise PSD. At low frequencies, the phase noise is typically limited by the flicker noise of the reference clock (which is proportional to the $f^{-3}$ part). In the plot of the frequencynoise PSD, this has a $f^{-1}$ roll-off, making it important to maximize $\omega_{\min }$, which could be resolved by using dynamical decoupling schemes, as they introduce an additional high-pass filtering [28,29,76,77]. The part of the phasenoise plot highlighted in red may be a source of concern [26], as it results in a frequency noise increasing as $f^{2}$ that exactly cancels the roll-off of the intrinsic qubit filter [Fig. 2(a)], thus resulting in a diverging integral for the fidelity (Table I) in the case in which no additional bandpass filtering is applied. However, the noise highlighted in red, visible in the phase-noise plot, originates from thermal noise added to the microwave signal by, e.g., the output driver of the microwave signal generator $[75,78]$. The additive noise, with generally a wide bandwidth, is more accurately modeled in the applied microwave magnetic field as follows:

$$
B(t)=\frac{2 \omega_{R}}{\gamma_{e}} \cos \left[\omega_{\mathrm{mw}} t+\phi+\phi_{n}(t)\right]+B_{\mathrm{add}}(t),
$$

where $B_{\text {add }}(t)$ represents the additive noise with PSD $S_{\text {add }}(\omega)$. The actual phase noise $\phi_{n}(t)$, indicated by the blue line in Fig. 3, is clearly band limited by the qubit-filter function due to the absence of the $f^{2}$ factor. The PSD of this additive noise has the same frequency dependence as the PSD of the phase noise [79]. The fidelity of the qubit
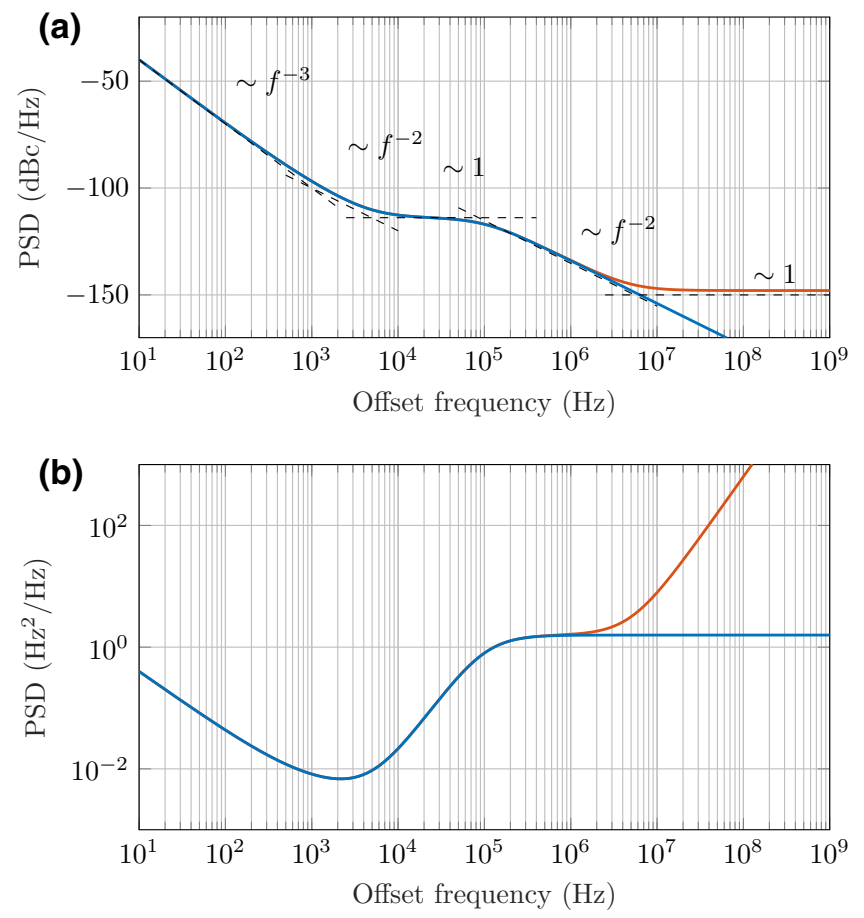

FIG. 3. A typical plot of (a) the phase noise and (b) the resulting frequency-noise PSD of a PLL-based frequency generator. The red line indicates the noise as measured by a phase-noise analyzer, whereas the blue line indicates the part of the noise that is actually phase noise. At high offset frequencies, where the lines diverge, wide-band additive noise shows up in the phasenoise plot, giving rise to a noise floor of around $-150 \mathrm{dBc} / \mathrm{Hz}$ in this example. 
operation in the presence of this type of noise is given in Table I.

Finally, the signal duration $T$ is also subject to random variations, i.e., jitter. However, since the period cannot vary during the operation, the noise in the timing can be simply treated considering the period jitter as a quasistatic error. This period jitter is determined by the single-sideband phase noise $S_{\phi}(\omega)$ of the reference clock (period $T_{\text {clk }}$ ) used to set the duration $[75,80]$ (Table I), following a highpass filter (HPF) characteristic with the corner frequency set by the duration $T$.

\section{B. Specifications for the idle operation and qubit-frequency multiplexing}

In a typical quantum algorithm, a qubit can be idle for a while, waiting for the operations on other qubits to finish, before being operated on, e.g., due to limitations in the hardware or data dependencies. This section discusses processes that cause the state of the qubit to degrade during an idle period lasting $T_{\text {nop }}$. The loss of the quantum state due to interactions with other qubits will be discussed in Sec. VB.

A qubit will perform an undesired $Z$ rotation (related to $T_{2}^{*}$ ) in the rotating frame if the microwave frequency is not matched to the qubit's Larmor frequency, even when the driving tone is not applied to the qubit. Evaluating the fidelity of an identity operation in the case of a frequency inaccuracy $\Delta \omega_{\mathrm{mw}, 0}$ leads to $F_{I_{\mathrm{nop}, \mathrm{mw}}}=1-\frac{1}{4} \Delta \omega_{\mathrm{mw}, 0}^{2} T_{\mathrm{nop}}^{2}$, which can easily be more stringent than the requirement due to a rotation (Table I).

Besides $Z$ rotations, unintended $X$ and $Y$ rotations of the qubit (related to $T_{1}$ ) are possible in the case in which power is present at the qubit's Larmor frequency. In general, a tone could be present at the qubit frequency, e.g., due to signal leakage from the microwave source or nonlinearities in the system leading to harmonic or intermodulation tones. The presence of a spurious tone that would give a Rabi frequency of $\omega_{\text {spur }}$ will reduce the fidelity as follows: $F_{I_{\text {nop,spur }}}=1-\frac{1}{4} \omega_{\text {spur }}^{2} T_{\text {nop. }}^{2}$.

Besides a tone, residual thermal noise could be present on the drive line. Considering a noise signal with spectral density $S_{R_{n}}(\omega)$, the fidelity is $F_{I_{\text {nop,noise }}}=1-$ $(1 / \pi) \int_{0}^{\infty} S_{R_{n}}(\omega)\left|H_{n}(\omega)\right|^{2} d \omega$, where

$$
\left|H_{n}(\omega)\right|^{2}=2 \frac{\sin ^{2}\left[\frac{T_{\text {nop }}}{2}\left(\omega-\omega_{0}\right)\right]}{\left(\omega-\omega_{0}\right)^{2}},
$$

which indicates that the noise spectrum is filtered by a sinc-shaped band-pass filter centered around $\omega_{0}$, with the following brick-wall approximation:

$$
\left|H_{n}(\omega)\right|^{2} \approx \begin{cases}T_{\mathrm{nop}}^{2} / 2, & \left|\omega-\omega_{0}\right| \leq \pi / T_{\mathrm{nop}} \\ 0, & \text { elsewhere. }\end{cases}
$$

Lastly, multiple qubits sharing the same control line, i.e., a single ESR line or control gates shorted together, can be controlled independently in the case in which each qubit has a unique Larmor frequency, as mentioned in Sec. II. This technique constitutes FDMA. However, when rotating a qubit with Larmor frequency $\omega_{0}$ by applying a microwave signal at frequency $\omega_{\mathrm{mw}}=\omega_{0}$, any unaddressed qubit on the same line with Larmor frequency $\omega_{0, \text { other }}=\omega_{0}+\omega_{0, \text { space }}$ will be affected. Similarly, even if not on the same drive line, another qubit could be unintentionally driven due to parasitic coupling such as capacitive or magnetic cross talk.

An expression for the fidelity of the unaddressed qubit with respect to the ideal identity operation is reported in the Supplemental Material [69] for a microwave pulse with a rectangular envelope [Fig. 4(a)] and it is plotted in Fig. 4(c), where we assume the same Rabi frequency $\omega_{R}$ for both qubits. As expected, driving the qubit with a larger amplitude (i.e., larger $\omega_{R}$ ) results in a shorter pulse for a given rotation angle, thus leading to a wider pulse bandwidth and, consequently, to a cross talk extending to qubits that are further away in frequency.

Although the expectation may arise that reducing the pulse bandwidth by proper engineering of the pulse envelope can lead to lower cross talk, Fig. 4(d) shows that also a Gaussian envelope [Fig. 4(b)] does not result in a much faster roll-off. As the figure shows, the fidelity can be limited by unintended $Z$ rotations of the unaddressed qubit. However, by applying a simple correction for the $Z$ rotation, the fidelity of the identity operation on the unaddressed qubit improves to the following:

$$
F_{I_{\mathrm{FDMA}}} \approx 1-\frac{\beta^{2}}{\alpha^{2}} \sin ^{2}\left(\frac{\theta}{2} \alpha\right) \geq 1-\frac{\beta^{2}}{\alpha^{2}}
$$

where $\alpha=\omega_{0, \text { space }} / \omega_{R}$ and $\beta=\omega_{R \text {,unaddressed }} / \omega_{R}$ and where, in general, the unaddressed qubit can have a different Rabi frequency $\left(\omega_{R \text {,unaddressed }}\right)$ at the same microwave amplitude, e.g., due to a lower coupling to the drive signal. As expected, the fidelity given by Eq. (8) is approximately proportional to the spectrum of the envelope of the applied pulse [Fig. 4(c)]. Consequently, reduction of the pulse bandwidth by proper engineering of the pulse envelope is an effective solution if the unintended $Z$ rotations are corrected, as shown in, e.g., Figs. 4(b) and 4(d), where a Gaussian envelope is employed.

A certain minimum frequency separation is necessary to achieve a target fidelity, as shown in Fig. 4(e) for the rectangular envelope. The lower bound on the fidelity as given in Eq. (8) is plotted as well, as the notches in the graph move depending on $\theta$. Similarly, if the coupling of the microwave drive is due to parasitic effects and is unwanted, a target fidelity for unaddressed qubits translates into a 
(a)

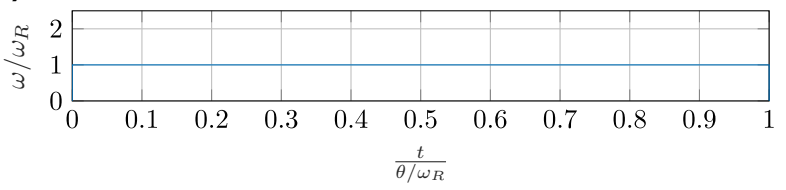

(c)

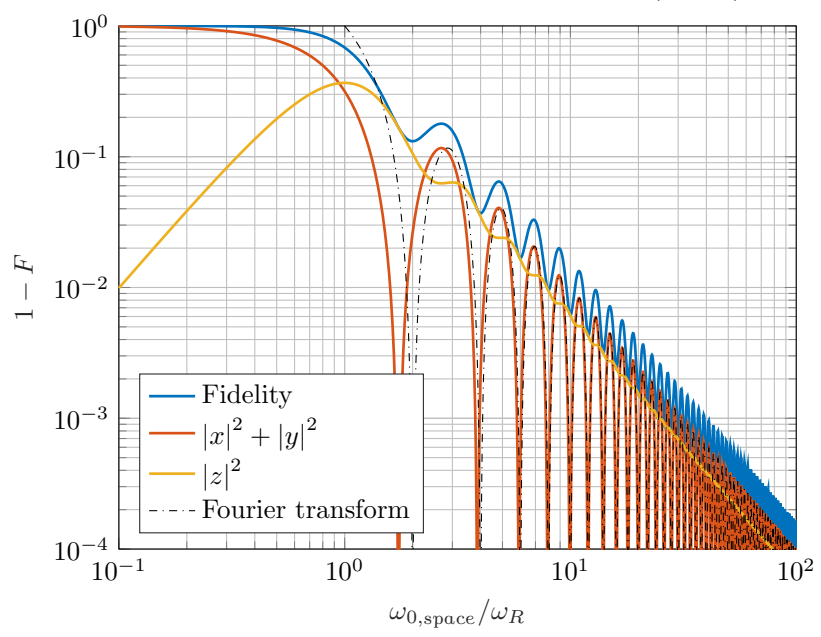

(e)

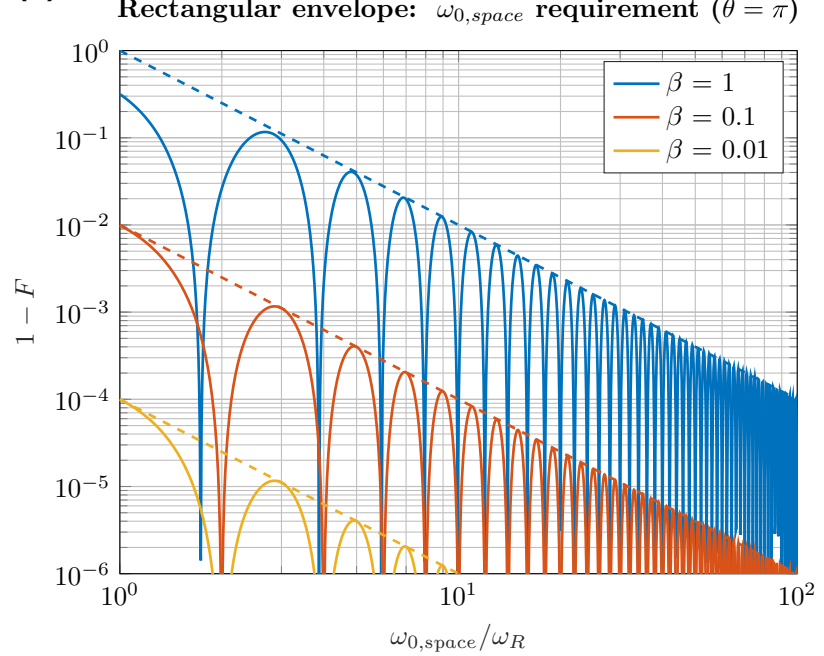

(b)

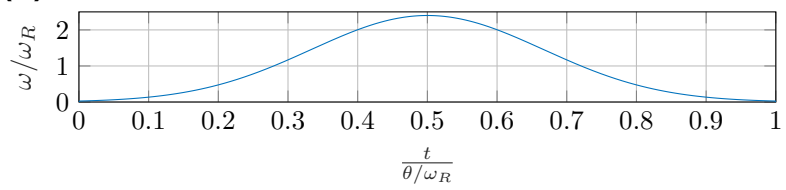

(d) FDMA with Gaussian envelope $(\theta=\pi)$

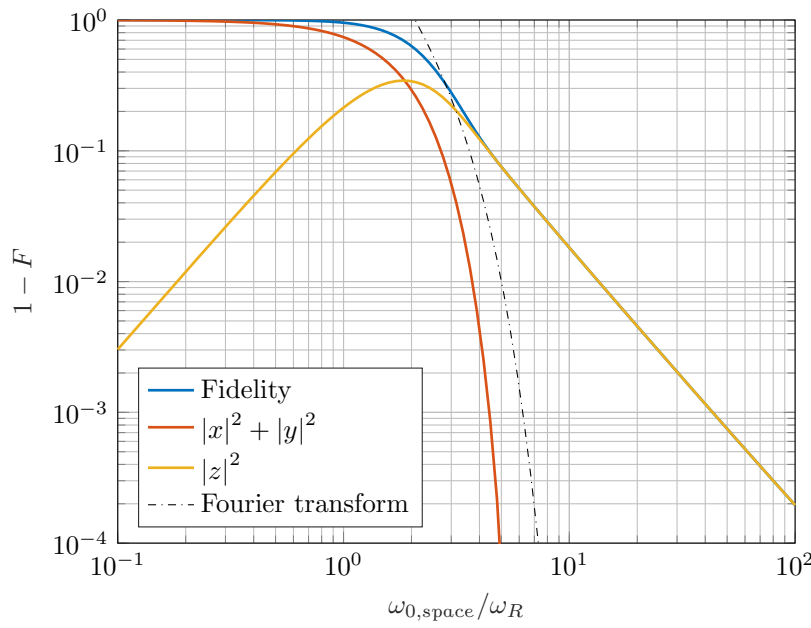

(f) Rectangular envelope: $\beta$ requirement $(\theta=\pi)$

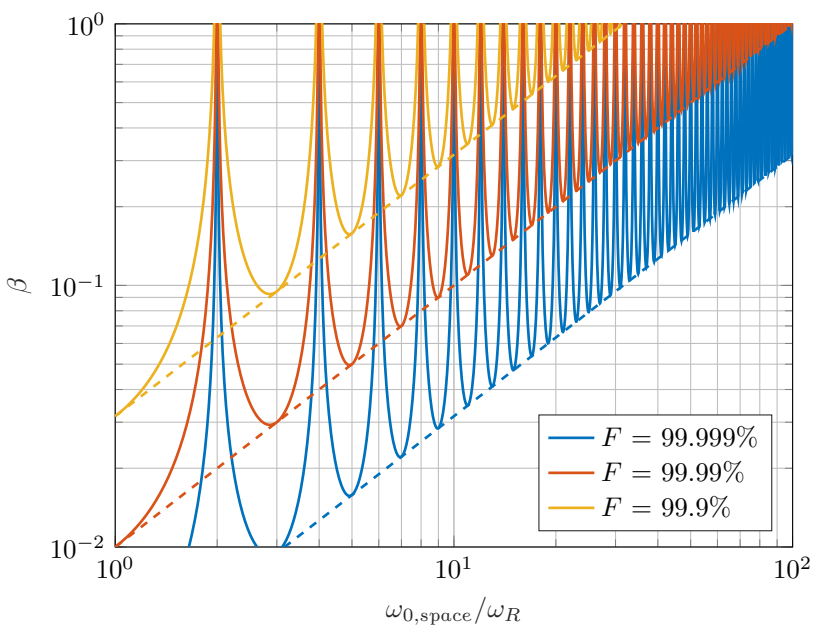

FIG. 4. Qubit-frequency multiplexing: the envelopes, achievable fidelity, and requirements in the case of a rectangular envelope. (a) The rectangular envelope under consideration. (b) The Gaussian envelope under consideration. (c) The infidelity of an identity operation (and the amount of $X$ or $Y$ rotation and $Z$ rotation) on a qubit spaced at $\omega_{0 \text {,space }}$ from the carrier for a rectangular envelope, along with the Fourier transform of the rectangular envelope. (d) The infidelity of an identity operation (and the amount of $X$ or $Y$ rotation and $Z$ rotation) on a qubit spaced at $\omega_{0, \text { space }}$ from the carrier for a Gaussian envelope, obtained by numerical simulation, along with the Fourier transform of the Gaussian envelope. (e) The frequency spacing required to achieve a certain fidelity at given relative signal strength $\beta$, for a rectangular envelope. The upper bound (dashed lines) is given in Eq. (8). (f) The driving tone attenuation $\beta$ required at a certain frequency spacing to achieve a given fidelity, for a rectangular envelope. The lower bound (dashed lines) is given in Eq. (9).

requirement in the driving tone attenuation [Fig. 4(f)]:

$$
\beta=\sqrt{1-F_{\text {corr }}} \frac{\alpha}{\left|\sin \left(\frac{\theta}{2} \alpha\right)\right|} \geq \sqrt{1-F_{\text {corr }}} \frac{\omega_{0, \text { space }}}{\omega_{R}} .
$$

Finally, FDMA has the potential to perform single-qubit gates on several qubits at the same time, using a single drive line. In that case, it is not sufficient to apply a compensating $Z$ rotation afterward, on another qubit, if that qubit is also performing an operation. As the $Z$ 
rotation is obtained gradually when an off-resonance tone is applied, the driving tone applied to perform the operation should be altered to compensate for this $Z$ rotation during the operation. This requires proper engineering of all the microwave pulses that are applied simultaneously [37,81-84].

\section{Case study of the specifications for a single-qubit operation}

With the information provided in Sec. IV, clear specifications for the control electronics can be derived. Table II shows as an example of how the total error budget can be allocated over the electronics specification to achieve a $99.9 \%$ fidelity for a $\pi$ rotation at a Rabi frequency of $1 \mathrm{MHz}$. The same fidelity is targeted for preserving the state of the qubit when not operating on it for a time equal to the operation time $\left(T_{\text {nop }}=T\right)$. The example considers the use of simple rectangular pulses, without any echo technique.

A Larmor frequency larger than $80 \mathrm{MHz}$ would be sufficient not to get impaired by fast-oscillating terms neglected by the rotating-wave approximation (RWA, see the Supplemental Material [69]). However, choosing $\omega_{0}=$ $10 \mathrm{GHz}$ is more in line with values used in practice and allows for a large qubit-frequency spacing. A frequency spacing of $1 \mathrm{GHz}$ is selected, the same as considered in the case study of two-qubit operations (Sec. VC). Such spacing is, however, approximately 10 times larger than required for minimizing the cross talk due to FMDA
(Sec. IV B). The example also shows the effect of the frequency noise as expected from isotopically purified $\mathrm{Si}-28$ (800 ppm ${ }^{29} \mathrm{Si}$ ), highlighting that its contribution to the infidelity is negligible in this example.

The values provided for the microwave amplitude assume a qubit plane based on EDSR, where an amplitude of $2 \mathrm{mV}$ at the gate is required for a Rabi frequency of $1 \mathrm{MHz}$ (close to the value reported in Ref. [44]). All specifications are valid at the gate, so that wiring attenuation and filtering might need to be factored in to refer the specifications back to the electronics.

Following these specifications, the microwave envelope (amplitude and duration) can be generated by, e.g., an AWG with a sample rate of at least 150 megasamples/s (MS/s), such that the sample time is less than $6.7 \mathrm{~ns}$, resulting in a maximum inaccuracy of $3.3 \mathrm{~ns}$. Furthermore, the AWG should have a resolution of 8 bits, such that at a fullscale swing of $4 \mathrm{mV}$, the quantization step is sufficiently low. To meet the noise requirement and the specifications on the residual driving when not operating the qubit ("Off spur" in Table II), an effective number of bits (ENOB) of only 6.5 bits is required.

The LO used for the up-conversion requires a frequency resolution of approximately $20 \mathrm{kHz}$ (for the inaccuracy). Assuming a $-20 \mathrm{~dB} / \mathrm{dec}$ slope of the phase noise, the single-side-band phase noise at $1 \mathrm{MHz}$ from the carrier, $\mathcal{L}(1 \mathrm{MHz})$, needs to be below $-106 \mathrm{dBc} / \mathrm{Hz}$. Furthermore, the LO's phase inaccuracy needs to be below $0.64^{\circ}$.

TABLE II. Example specifications for the control electronics for single-qubit operations. The PSD values provided as a comment assume a white spectrum for the amplitude and frequency noise (i.e., $-20 \mathrm{~dB} / \mathrm{dec}$ for the phase noise).

\begin{tabular}{|c|c|c|c|c|}
\hline & \multirow[b]{2}{*}{ Value } & \multicolumn{2}{|c|}{ Infidelity contribution } & \multirow[b]{2}{*}{ Comment } \\
\hline & & To an operation & To idling & \\
\hline \multicolumn{5}{|l|}{ Frequency } \\
\hline Nominal & $10 \mathrm{GHz}$ & $0.64 \times 10^{-9}$ & & RWA when driving a qubit \\
\hline Spacing & $1 \mathrm{GHz}$ & & $1 \times 10^{-6}$ & FDMA leakage with rectangular envelopes \\
\hline Inaccuracy & $11 \mathrm{kHz}$ & $125 \times 10^{-6}$ & $308 \times 10^{-6}$ & \\
\hline Oscillator noise & $11 \mathrm{kHz}_{\mathrm{rms}}$ & $125 \times 10^{-6}$ & $308 \times 10^{-6}$ & $\mathrm{ENBW}=2.5 \mathrm{MHz}, \mathcal{L}(1 \mathrm{MHz})=-106 \mathrm{dBc} / \mathrm{Hz}$ \\
\hline Nuclear spin noise & $1.9 \mathrm{kHz}_{\mathrm{rms}}$ & $3.6 \times 10^{-6}$ & $8.9 \times 10^{-6}$ & From Ref. [45], $T_{2}^{*}=120 \mu \mathrm{s}$ \\
\hline Wide-band noise & $12 \mu \mathrm{V}_{\mathrm{rms}}$ & $125 \times 10^{-6}$ & & $\mathrm{ENBW}=2.9 \mathrm{MHz}, S_{\mathrm{add}}=7.1 \mathrm{nV} / \sqrt{\mathrm{Hz}}$ \\
\hline \multicolumn{5}{|r|}{ 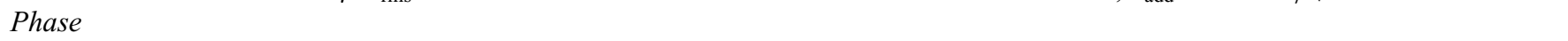 } \\
\hline Inaccuracy & $0.64^{\circ}$ & $125 \times 10^{-6}$ & $31 \times 10^{-6}$ & FDMA $Z$ corrections limit the no operation \\
\hline \multicolumn{5}{|l|}{ Amplitude } \\
\hline Nominal & $2 \mathrm{mV}$ & & & Full scale: $4 \mathrm{mV}$, rms: $1.4 \mathrm{mV}_{\mathrm{rms}}$ \\
\hline Inaccuracy & $14 \mu \mathrm{V}$ & $125 \times 10^{-6}$ & & \\
\hline Noise & $14 \mu \mathrm{V}_{\mathrm{rms}}$ & $125 \times 10^{-6}$ & & $\mathrm{ENBW}=1.0 \mathrm{MHz}, \mathrm{PSD}=14 \mathrm{nV} / \sqrt{\mathrm{Hz}}, \mathrm{SNR}=-40 \mathrm{~dB}$ \\
\hline Off spur & $19 \mu \mathrm{V}$ & & $217 \times 10^{-6}$ & $-41 \mathrm{dBc}$ \\
\hline Off noise & $10 \mu \mathrm{V}_{\mathrm{rms}}$ & & $125 \times 10^{-6}$ & $\mathrm{ENBW}=2.0 \mathrm{MHz}, \mathrm{PSD}=7.1 \mathrm{nV} / \sqrt{\mathrm{Hz}}$ \\
\hline \multicolumn{5}{|l|}{ Duration } \\
\hline Nominal & $500 \mathrm{~ns}$ & & & \\
\hline Inaccuracy & $3.6 \mathrm{~ns}$ & $125 \times 10^{-6}$ & & \\
\hline \multirow[t]{2}{*}{ Noise } & $3.6 \mathrm{~ns}_{\mathrm{rms}}$ & $125 \times 10^{-6}$ & & \\
\hline & & $F_{X, Y}=99.9 \%$ & $F_{I}=99.9 \%$ & \\
\hline
\end{tabular}


(a)

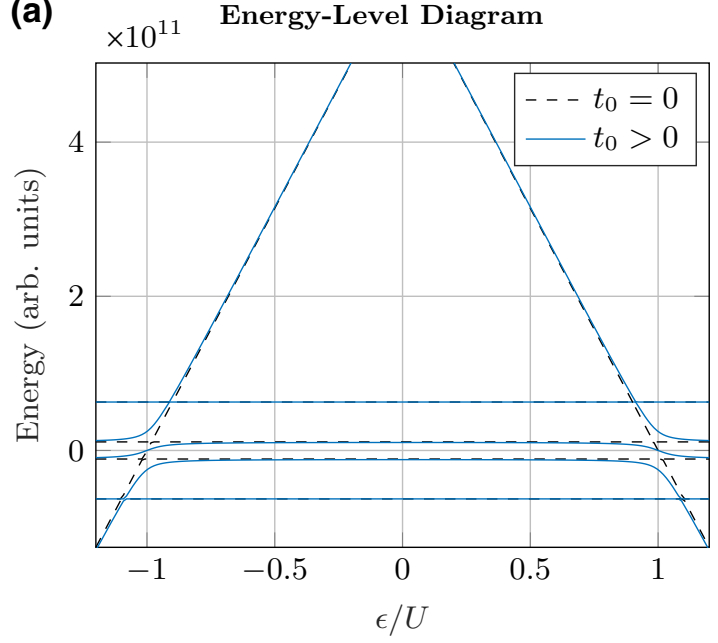

(b) The operation speed $\omega_{o p}\left(U=1000 t_{0 n}\right)$

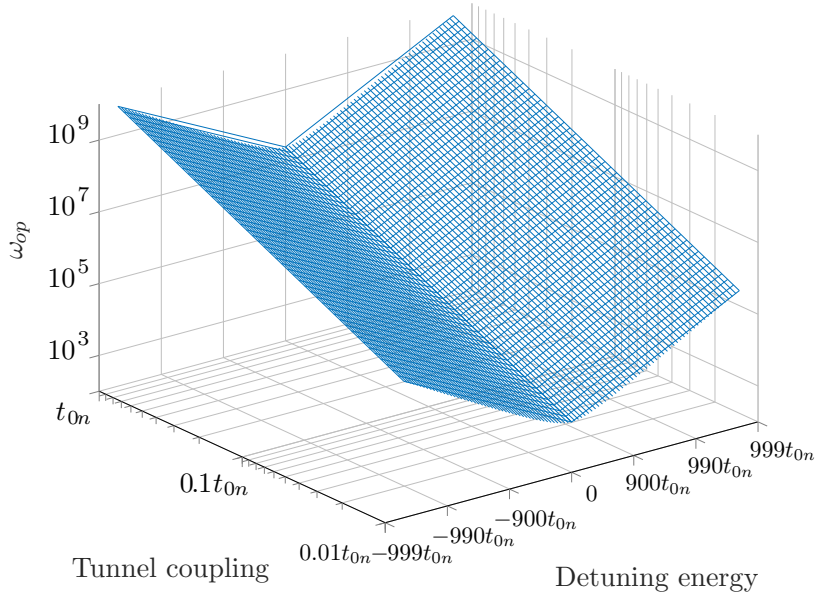

FIG. 5. (a) The energy-level diagram of the two-qubit system. An avoided crossing is visible for $|\epsilon|=U$ when there is a finite tunnel coupling between the dots. (b) The two-qubit operation speed $\omega_{\mathrm{op}}$ [Eq. (11)] versus the interdot tunnel coupling and detuning. A nominal tunnel coupling $t_{0 n}$ of $1 \mathrm{GHz}$ is used.

\section{SIGNAL SPECIFICATIONS FOR TWO-QUBIT OPERATIONS}

\section{A. Fidelity of a two-qubit operation}

As stated in Sec. II, by default the tunnel coupling between the qubits is negligible and the qubits have the same potential, i.e., they are not detuned. By increasing the tunnel coupling and/or by detuning the qubits, the qubit interaction increases and a two-qubit gate can be obtained. In this system, by leveraging this exchange interaction, a two-qubit exchange gate and a $C$ phase gate can be implemented. With either of these gates and single-qubit operations, a universal set is obtained.

To describe the physical interactions required for the two-qubit gate, higher energy levels need to be modeled in the Hamiltonian. The analysis presented here is limited to the interaction between two neighboring qubits, $A$ and $B$, and to the single-dot singlet states $(|0,2\rangle$ represents the singlet state in the right dot and $|2,0\rangle$ the singlet state in the left). In the basis $\Psi=$ $[|\uparrow, \uparrow\rangle,|\uparrow, \downarrow\rangle,|\downarrow, \uparrow\rangle,|\downarrow, \downarrow\rangle,|0,2\rangle,|2,0\rangle]$, the Hamiltonian of a double quantum dot is given by the following $(\hbar=1)[55,85,86]$ :

$$
H=\left[\begin{array}{cccccc}
-\omega_{0} & 0 & 0 & 0 & 0 & 0 \\
0 & \frac{\delta \omega_{0}}{2} & 0 & 0 & t_{0} & t_{0} \\
0 & 0 & -\frac{\delta \omega_{0}}{2} & 0 & -t_{0} & -t_{0} \\
0 & 0 & 0 & \omega_{0} & 0 & 0 \\
0 & t_{0} & -t_{0} & 0 & U-\epsilon & 0 \\
0 & t_{0} & -t_{0} & 0 & 0 & U+\epsilon
\end{array}\right]
$$

where $\omega_{0}=\left(\omega_{0, A}+\omega_{0, B}\right) / 2, \delta \omega_{0}=\omega_{0, B}-\omega_{0, A}$, and $\omega_{0, A}$ and $\omega_{0, B}$ are the Larmor frequencies of the two qubits. The charging energy $(U)$ is assumed to be the same for both dots. The tunnel coupling between the quantum dots $\left(t_{0}\right)$ has an exponential relation to the voltage on the barrier gate and the detuning energy $(\epsilon)$ is controlled by the voltage difference on the plunger gates of the dots $\left(V_{d}\right)$ via the lever arm $\alpha=\Delta \epsilon / \Delta V_{d}$.

An avoided crossing is observed in the energy-level diagram for $|\epsilon|=U$ and $t_{0}>0$ [Fig. 5(a)], which gives rise to eigenenergies that are different from the case of two isolated dots $\left(t_{0} \sim 0\right)$ for any detuning. This change of eigenenergy and the corresponding eigenstate form the basis of the two-qubit operations. An investigation of the eigenenergies of the Hamiltonian in Eq. (10) reveals that the total change in eigenenergy equals the following $(\hbar=1)$ :

$$
\omega_{\mathrm{op}}=4 t_{0}^{2} \frac{U}{U^{2}-\epsilon^{2}}
$$

Note that the expression used in this paper for $\omega_{\text {op }}$ derives directly from the Hamiltonian of Eq. (10). However, experiments have reported $\omega_{\text {op }}$ as an exponential function of detuning [87].

As $\omega_{\text {op }}$ describes the amount of exchange interaction, it directly sets the speed of the two-qubit operation. A plot of $\omega_{\mathrm{op}}$ versus the tunnel coupling and detuning is shown in Fig. 5(b). To perform the two-qubit operation, a control pulse must be applied, to move the system away from the default point (negligible tunnel coupling and zero detuning) to the desired operating point, where there is sufficient exchange interaction such that a two-qubit operation is performed. From Fig. 5(b), it is clear that a fast gate can be 
obtained at finite detuning, becoming faster closer to the avoided crossing, controlled by the detuning and/or the tunnel coupling. Alteratively, operation at zero detuning (the charge-symmetry point [30]) is possible, controlled by the tunnel coupling alone. Depending on whether the control parameter, the detuning, and/or the tunnel coupling is changed adiabatically or diabatically, a $C$ phase or exchange gate, or a mixture of the two, is obtained.

In the case in which the control parameter changes slowly, i.e., adiabatically, the resulting operation, in the rotating frame, can be described by the following diagonal matrix:

$$
U_{C Z}(t)=\operatorname{diag}\left(1, e^{-i \phi_{Z, A}}, e^{-i \phi_{Z, B}}, 1\right),
$$

where $\phi_{Z, A}$ and $\phi_{Z, B}$ are the acquired phases in the rotating frame. Two additional $Z$ rotations with angles $\phi_{Z, A}$ and $\phi_{Z, B}$ can be applied to the right and left qubit, respectively, to obtain the $C$ phase gate with $\theta_{C Z}=-\left(\phi_{Z, A}+\right.$ $\left.\phi_{Z, B}\right)=\omega_{\mathrm{op}} t$. These $Z$ rotations can easily be obtained by updating the software reference frame $[37,38]$. In the case in which $\theta_{C Z}=\pi$, a controlled- $Z$ operation is obtained. Interestingly, the total acquired phase $\left(\phi_{Z, A}+\phi_{Z, B}\right)$ is independent of $\delta \omega_{0}$. However, when $\delta \omega_{0}=\sqrt{2} t_{0}, \phi_{Z, A}=\phi_{Z, B}$ $[55,85,86]$, whereas for $\delta \omega_{0}=0, \phi_{Z, A}=0$.

If, instead, the control parameter is changed rapidly, i.e., diabatically, and the Larmor-frequency difference is negligible $\left(\delta \omega_{0} \ll \omega_{\mathrm{op}}\right)$, the resulting operation, in the rotating frame, is as follows:

$$
U_{J}(t) \approx\left[\begin{array}{cccc}
1 & 0 & 0 & 0 \\
0 & \frac{1+e^{i \theta_{J}}}{2} & \frac{1-e^{i \theta_{J}}}{2} & 0 \\
0 & \frac{1-e^{i \theta_{J}}}{2} & \frac{1+e^{i \theta_{J}}}{2} & 0 \\
0 & 0 & 0 & 1
\end{array}\right],
$$

where $\theta_{J}=\omega_{\mathrm{op}} t$. In the case in which $\theta_{J}=\pi$, a SWAP operation is obtained. Note that since for an accurate exchange operation the Larmor-frequency difference should be sufficiently small, the possibility of using FDMA for single-qubit operations (Sec. IV B) is limited.

From Eq. (10) it follows that the two-qubit operations are affected by the Larmor frequencies $\left(\omega_{0, A}, \omega_{0, B}\right)$, the tunnel coupling $\left(t_{0}\right)$, the charging energy $(U)$, and the detuning $(\epsilon)$. Furthermore, the operation depends on the total duration $(T)$ for which the two-qubit gate is active. The effect of errors, both static and dynamic, on the fully electrically controlled parameters $\left(t_{0}, \epsilon\right.$, and $\left.T\right)$ is analyzed in the subsequent section. Detailed derivations of the formulas can be found in the Supplemental Material [69].

The resulting fidelity in the case of control signal inaccuracies is summarized in Table III for the exchange gate and the $C$ phase gate, both at zero detuning and finite detuning. For the exchange gate, we assume that no Larmorfrequency difference between the qubits exists, since for

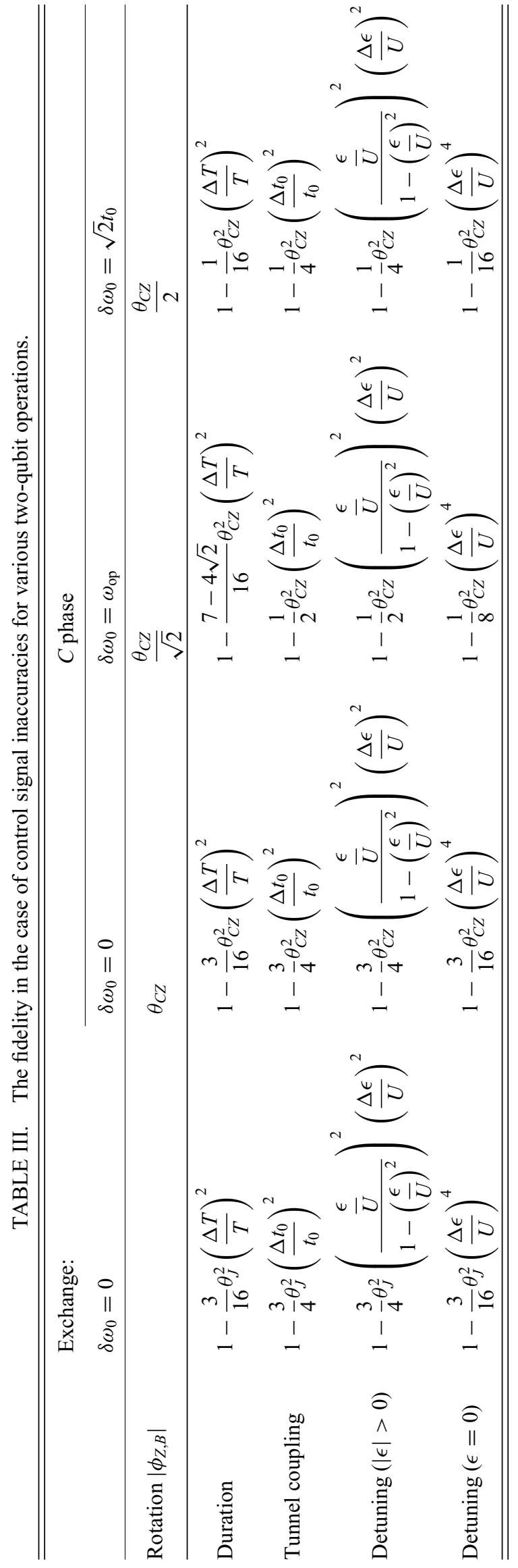


such a gate $\delta \omega_{0} \ll \omega_{\text {op }}$ is required, while for the $C$ phase gate, various scenarios are analyzed $\left(\delta \omega_{0}=0, \delta \omega_{0}=\omega_{\mathrm{op}}\right.$, and $\left.\delta \omega_{0}=\sqrt{2} t_{0}\right)$. In the table, $T$ denotes the qubit gate operation time and inaccuracies are shown with the prefix $\Delta$. The table provides values for different rotation angles $\left(\theta_{C Z}, \theta_{J}\right)$. In the case of the $C$ phase gate, additional $Z$ rotations might be required, which only have a very small effect on the fidelity [Eq. (3)].

The error contributions have a quadratic relation with the infidelity except for detuning errors for $\epsilon=0$, where a fourth-order dependence is found. This implies an improved robustness to detuning errors when operating at the charge-symmetry point $(\epsilon=0)[30,31]$.

For low-frequency variations, i.e., those changing over a time scale longer than the operation time, the same approach as for single-qubit operations holds and the expected fidelity follows the same equations as given in Table III when replacing the inaccuracy, such as $\Delta \epsilon$, with the standard deviation of the variation (assuming a Gaussian distribution). An exception is for detuning errors when operating at the charge-symmetry point $[30,31]$, because of the fourth-order dependence. For a static but random error $\Delta$ for which $F=1-c \Delta^{4}$, the expected fidelity is $F=1-3 c \sigma^{4}$, if $\Delta$ follows a Gaussian distribution with standard deviation $\sigma$ and zero mean. Consequently, there is a slightly higher sensitivity to noise than to static errors for the detuning.

For timing variations, only the total duration matters and high-frequency noise is filtered as described in Table I. Moreover, similar to the single-qubit gate, numerical simulations of the Hamiltonian have shown sensitivity to high-frequency noise $\left(>\omega_{\mathrm{op}}\right)$ only in a pass band with a bandwidth that is inversely proportional to the operation duration, for both the electrically controlled detuning energy and tunnel coupling in the case of the two-qubit gate (see the Supplemental Material [69]). The quantum state is, however, also affected by noise around the frequencies corresponding to the allowed energy transitions, in a pass band with a similar bandwidth. Consequently, it is important that the high-frequency noise components in the signals applied to the barrier gates and plunger gates are properly filtered. However, since the exchange gate requires a diabatic change in the control parameter, only limited filtering can be applied. Closed-form analytical expressions for these effects have not been studied.

\section{B. Specifications for the idle operation}

Since, in practice, the tunnel coupling cannot be fully removed, the two-qubit operation is never completely turned off. The interaction strength can, however, be slowed down significantly, thus leading to a fidelity with respect to the ideal identity operation for the exchange and
$C$ phase gates as follows:

$$
F_{I_{\mathrm{nop}, J}}= \begin{cases}1-\frac{3}{16} \omega_{\mathrm{op}, \mathrm{off}}^{2} T_{\mathrm{nop}}^{2}, & \delta \omega_{0}=0, \\ 1-\frac{7-4 \sqrt{2}}{16} \omega_{\mathrm{op}, \mathrm{off}}^{2} T_{\mathrm{nop}}^{2}, & \delta \omega_{0}=\omega_{\mathrm{op}}, \\ 1-\frac{1}{16} \omega_{\mathrm{op}, \mathrm{off}}^{2} T_{\text {nop }}^{2}, & \delta \omega_{0}=\sqrt{2} t_{0},\end{cases}
$$

where $\omega_{\mathrm{op}, \text { off }}$ is the reduced interaction strength during the time $T_{\text {nop }}$ when no operation is applied.

Following Eq. (11), the interaction strength can be reduced by lowering the tunnel coupling while not changing the detuning. A two-qubit operation performed at finite detuning could also be controlled using only the detuning. However, assuming that the interaction is considered to be off at zero detuning, the operation might need to be performed at far detuning. As mentioned before, operating closer to the avoided crossing reduces the tolerance to inaccuracies and noise in the detuning (Table III).

\section{Case study of the specifications for a two-qubit operation}

Specifications for the control electronics responsible for the two-qubit operation can be derived using the results presented in Sec. V. This example develops on the example given in Sec. IV C and, for instance, assumes that the same oscillator is used to keep the coherence with the qubits. Two examples will be given here, one at zero detuning and one at finite detuning. Both focus on the $C$ phase gate, operating at $\delta \omega_{0}=\sqrt{2} t_{0}$. This choice for the Larmorfrequency difference gives the most relaxed specifications for the control electronics, while at smaller $\delta \omega_{0}$, the specifications can be up to $\sqrt{3}$ times more demanding (see Table III).

The Larmor-frequency difference is chosen as $1 \mathrm{GHz}$, to achieve a two-qubit operation speed of $\omega_{\mathrm{op}}=2 \mathrm{MHz}$ at zero detuning, while maintaining $\delta \omega_{0}=\sqrt{2} t_{0}$ [Eq. (11)]. Example specifications for this operation are given in Table IV. To further increase the operating speed, an even higher qubit-frequency spacing would be required or $\delta \omega_{0}<\sqrt{2} t_{0}$. Alternatively, the operating speed can be enhanced to, e.g., $20 \mathrm{MHz}$, by operating the $C$ phase gate at finite detuning [Eq. (11)], as shown in another example (Table IV).

Both examples target a fidelity of $99.9 \%$ for a $C$ phase gate with $\theta_{C Z}=\pi$. The examples also indicate the specifications required for idling two qubits at $99.9 \%$ fidelity for a duration of $500 \mathrm{~ns}$, the same as for the example in Sec. IV C.

For the charging energy and tunnel coupling, typical values are chosen. As the relation to the gate voltage is device dependent, no values for the required electrical specifications are given. Note that in either example, the tunnel coupling only has to change by a factor of approximately 
TABLE IV. Example specifications for the control electronics when operating a $C$ phase gate at zero detuning and at finite detuning. The PSD values provided assume a white spectrum with an ENBW of approximately $10 \mathrm{MHz}\left(\omega_{\mathrm{op}}=2 \mathrm{MHz}\right)$ when operating at zero detuning and an ENBW of approximately $100 \mathrm{MHz}\left(\omega_{\mathrm{op}}=20 \mathrm{MHz}\right)$ at finite detuning. A nominal charging energy of $83 \mathrm{mV}(4.1$ $\mathrm{meV}, 1.0 \mathrm{THz}$ ) is assumed.

\begin{tabular}{|c|c|c|c|c|c|}
\hline & \multicolumn{2}{|c|}{ Value } & \multicolumn{3}{|c|}{ Infidelity contribution to } \\
\hline & $\epsilon=0$ & $\epsilon>0$ & $\begin{array}{l}\text { An operation } \\
\epsilon=0\end{array}$ & $\begin{array}{l}\text { An operation } \\
\epsilon>0\end{array}$ & Idling \\
\hline \multicolumn{6}{|l|}{ Frequency } \\
\hline Spacing & $1 \mathrm{GHz}$ & $1 \mathrm{GHz}$ & & & \\
\hline Inaccuracy & $11 \mathrm{kHz}$ & $11 \mathrm{kHz}$ & $77 \times 10^{-6}$ & $0.8 \times 10^{-6}$ & $308 \times 10^{-6}$ \\
\hline Oscillator noise & $11 \mathrm{kHz}_{\mathrm{rms}}$ & $11 \mathrm{kHz}_{\mathrm{rms}}$ & $77 \times 10^{-6}$ & $0.8 \times 10^{-6}$ & $308 \times 10^{-6}$ \\
\hline Nuclear-spin noise & $1.9 \mathrm{kHz}_{\mathrm{rms}}$ & $1.9 \mathrm{kHz}_{\mathrm{rms}}$ & $2.2 \times 10^{-6}$ & $0.02 \times 10^{-6}$ & $8.9 \times 10^{-6}$ \\
\hline \multicolumn{6}{|l|}{ Duration } \\
\hline Nominal & $250 \mathrm{~ns}$ & $25 \mathrm{~ns}$ & & & \\
\hline Error & $5.3 \mathrm{~ns}$ & $0.58 \mathrm{~ns}$ & $281 \times 10^{-6}$ & $333 \times 10^{-6}$ & \\
\hline \multicolumn{6}{|l|}{ Detuning energy } \\
\hline Nominal & $0 \mathrm{mV}(0 \mu \mathrm{eV}, 0 \mathrm{GHz})$ & $78 \mathrm{mV}(3.9 \mathrm{meV}, 0.95 \mathrm{THz})$ & & & \\
\hline Error & $\begin{array}{l}12 \mathrm{mV}(0.60 \mathrm{meV}, 0.15 \mathrm{THz}) \\
\sigma=9.2 \mathrm{mV}_{\mathrm{rms}} \\
\mathrm{PSD}=2.9 \mu \mathrm{V} / \sqrt{\mathrm{Hz}}\end{array}$ & $\begin{array}{l}0.10 \mathrm{mV}(5.1 \mu \mathrm{eV}, 1.2 \mathrm{GHz}) \\
\sigma=0.10 \mathrm{mV}_{\mathrm{rms}} \\
\mathrm{PSD}=10 \mathrm{nV} / \sqrt{\mathrm{Hz}}\end{array}$ & $281 \times 10^{-6}$ & $333 \times 10^{-6}$ & \\
\hline \multicolumn{6}{|l|}{ Tunnel coupling } \\
\hline Nominal & $0.71 \mathrm{GHz}(2.9 \mu \mathrm{eV})$ & $0.71 \mathrm{GHz}(2.9 \mu \mathrm{eV})$ & & & \\
\hline Error & $7.5 \mathrm{MHz}(31 \mathrm{neV})$ & $8.2 \mathrm{MHz}(34 \mathrm{neV})$ & $281 \times 10^{-6}$ & $333 \times 10^{-6}$ & \\
\hline \multirow[t]{2}{*}{ Off value } & $78 \mathrm{MHz}(0.32 \mu \mathrm{eV})$ & $78 \mathrm{MHz}(0.32 \mu \mathrm{eV})$ & & & $374 \times 10^{-6}$ \\
\hline & & & $F_{C Z}=99.9 \%$ & $F_{C Z}=99.9 \%$ & $F_{I}=99.9 \%$ \\
\hline
\end{tabular}

9 to turn the operation on or off. In the case of operation at finite detuning, this assumes that zero detuning is applied when the operation is turned off.

The detuning energy is directly related to the voltage on the plunger gate via the lever arm, for which a typical value of $\alpha=0.05 \mathrm{eV} / \mathrm{V}$ is assumed [44]. When operating at finite detuning, the detuning energy is chosen at $95 \%$ of the charging energy. Even though higher operating speeds can be obtained by moving even closer to the avoided crossing, the electrical specifications become increasingly challenging. When operating at the charge-symmetry point, very large detuning errors can be tolerated (at which point the approximations used to derive the expressions in Table III do not hold any more). When operating at moderate detuning, the error specification for the detuning is more than 100 times stricter. Moreover, as the operation at finite detuning is faster with the same tunnel coupling, the signal bandwidth must be larger, with a larger noise bandwidth. As a rough estimate, the ENBW has been chosen as 5 times the operating speed in both examples, which seems plausible as an adiabatic change is required (for the exchange gate, the situation might be worse). As a result, the maximum allowed noise spectral density, assuming white noise, is much lower. For the given example, this results in a difference of almost 5 orders of magnitude in the noise PSD.

In the example operation at finite detuning, the detuning control can be achieved by an AW running at a sample rate of 1 gigasamples/s $(\mathrm{GS} / \mathrm{s})$ for a maximum timing inaccuracy of $0.5 \mathrm{~ns}$. Assuming that the AWG has to cover a voltage range of $-U \cdots U$ (where $U$ is the charging energy), it must have a 10-bit resolution to meet the accuracy specification of the detuning energy.

\section{SIGNAL SPECIFICATIONS FOR QUBIT READOUT}

\section{A. Fidelity for qubit readout}

For the readout of the quantum state, the Pauli spinblockade readout [88] is analyzed, since it offers several advantages with respect to the other possible alternative, i.e., the Elzerman readout [65]: no electron reservoir is required next to the quantum dot; and the Zeeman energy splitting does not have to be much higher than the thermal energy, thus enabling operation at higher temperatures and/or lower Larmor frequencies. As a drawback, the Pauli spin-blockade readout involves two quantum dots, where the measurement involves discrimination between the singlet and triplet states.

Even though relaxation, which is quantified by the relaxation time $T_{1}$, is an important limiting factor in qubit readout, its effect is not considered in the following analysis, as all gates are assumed to be performed in a time significantly smaller than $T_{1}$. Furthermore, in our analysis, we assume that the spin-dependent charge state resulting from a Pauli spin-blockade readout is measured using a charge sensor. As a result, the readout fidelity is determined by various factors: 
(a) $P_{\text {charge }}$ : the probability that the spin state is correctly projected to the charge state.

(b) $P_{\text {sense }}$ : the probability that the charge sensor correctly detects the charge state.

(c) $P_{\text {detect }}$ : the probability that the readout circuit correctly discriminates the signal of the charge sensor.

The overall readout fidelity is then as follows:

$$
F_{\text {read }} \approx P_{\text {charge }} \times P_{\text {sense }} \times P_{\text {detect }} .
$$

The probability $P_{\text {sense }}$ is limited by, e.g., interference on one of the charge-sensor bias gates and charge noise in the substrate. As this depends highly on the type of sensor employed and the sensor integration, this error contribution will not be discussed further.

The quantum-dot control electronics limit $P_{\text {charge, }}$ as discussed in Sec. VIB, while the readout electronics limit $P_{\text {detect }}$, as discussed in Sec. VI C.

\section{B. Specifications for the electronics controlling the spin to charge conversion}

For the analysis of the charge transfer in the Pauli spinblockade readout, the Hamiltonian of Eq. (10) is extended with the lowest-energy triplet states (either due to the valley splitting or the orbital energy splitting). Those states are spaced by a singlet-triplet energy splitting $E_{\mathrm{ST}}$ from the singlet energy level (for the Hamiltonian, see the Supplemental Material [69]). A plot of the energy of the stationary states versus the detuning near the avoided crossing is shown in Fig. 6(a). For the following discussion, only the $|\downarrow, \downarrow\rangle$ and $|\downarrow, \uparrow\rangle$ states, highlighted in Fig. 6(a), need to be considered.

The Pauli spin-blockade readout relies on $E_{\mathrm{ST}}$ for the discrimination of the single-dot singlet configuration from three possible single-dot triplet configurations. Considering a pair of neighboring qubits, the state of the right qubit can be measured as follows. The left qubit is initialized in the $|\downarrow\rangle$ state. By detuning adiabatically to a point between the singlet avoided crossing and the triplet avoided crossing (with $t_{0}>0$ ), only the $|\downarrow, \uparrow\rangle$ state (at $\epsilon=0$ ) becomes a singlet and both electrons will move into the same dot. This charge movement can be measured using a charge sensor. Based on the measurement result, it is then clear whether the qubits are in a singlet or one of the three triplet configurations. This scenario is analyzed here.

Starting from the $|\downarrow, \downarrow\rangle$ state, there is a small probability $P(\operatorname{transfer}|| \downarrow, \downarrow\rangle)$ that both electrons will end up in the same dot. Similarly, starting from the $|\downarrow, \uparrow\rangle$ state, there is a small probability $P$ (no transfer ||$\downarrow, \uparrow\rangle$ ) that no charge will transfer. The probability of a correct spin to charge conversion can be defined as follows:

$$
\left.\left.P_{\text {charge }}=1-P(\text { transfer }|| \downarrow, \downarrow\rangle\right)-P(\text { no transfer }|| \downarrow, \uparrow\rangle\right) .
$$

(a)

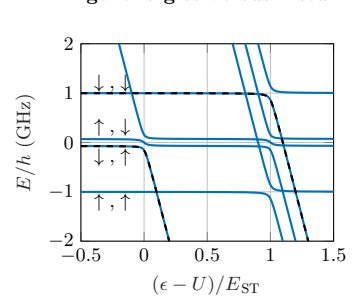

(c)

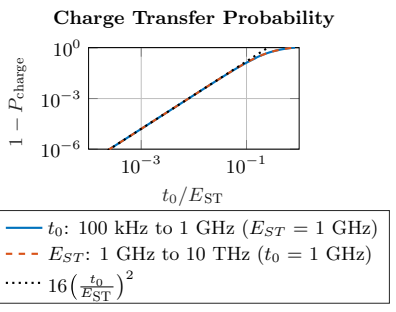

(b)

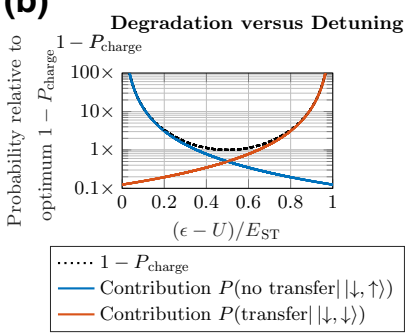

(d)

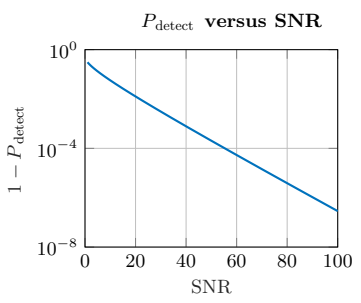

(e)

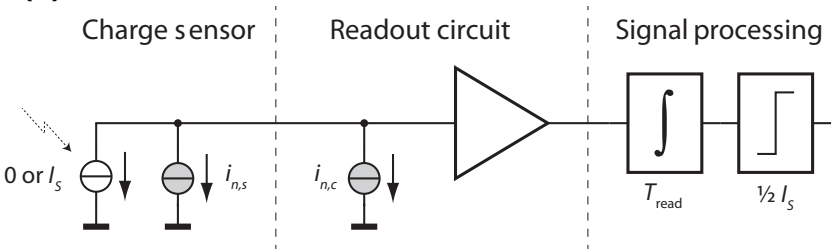

FIG. 6. (a) The energy of the stationary states versus the detuning near the avoided crossing. The black dashed lines indicate the states where the left qubit was originally in the $|\downarrow\rangle$ state. (b) The error probability $1-P_{\text {charge }}$, along with the individual error contributors, at various points of detuning as simulated for various tunnel rates, Larmor frequencies, charging energies, and singlet-triplet energy splittings (each varied over a decade; the resulting plots are overlapping). The obtained probabilities are plotted relative to the optimum, i.e., the lowest error probability at $\epsilon=U+E_{\mathrm{ST}} / 2$, thereby showing the degradation when moving away from the optimum detuning. (c) The simulated probability $1-P_{\text {charge }}$ versus the singlet-triplet splitting, normalized to the tunnel coupling at $\epsilon=U+E_{\mathrm{ST}} / 2$, while sweeping either the tunnel coupling or the singlet-triplet energy splitting. (d) A plot of $1-P_{\text {detect }}$ versus SNR in the case of Gaussiandistributed noise. (e) A model of a typical readout chain, showing the sensor, the readout electronics, and the required signal processing for the measurement discrimination. Additional sources modeling the noise are shown in gray.

The analysis is again simplified by assuming an ideal adiabatic change in the detuning energy. The results presented in this section are obtained from numerical simulations of the Hamiltonian.

Simulations show that the highest $P_{\text {charge }}$ is obtained by detuning to $\epsilon=U+E_{\mathrm{ST}} / 2$, i.e., equidistant between the singlet and triplet avoided crossings, as shown in Fig. 6(b). The shape of the probability versus $(\epsilon-U) / E_{\mathrm{ST}}$ plot is independent of the Larmor frequency, assuming that $\omega_{0} \ll$ $E_{\mathrm{ST}}$. Although the shape remains the same, the obtainable 
TABLE V. Example specifications for the control electronics. The PSD values provided assume a white spectrum with an ENBW of approximately $1 \mathrm{MHz}$ for the detuning control and a measurement time of $T_{\text {read }}=0.6 \mu \mathrm{s}$. A nominal charging energy of $82.7 \mathrm{mV}(4.1$ $\mathrm{meV}, 1.0 \mathrm{THz})$ is assumed and a singlet-triplet energy splitting of $1.0 \mathrm{mV}(50 \mu \mathrm{eV}, 12 \mathrm{GHz})$.

\begin{tabular}{|c|c|c|c|}
\hline & Value & \multicolumn{2}{|c|}{ Infidelity contribution to the readout } \\
\hline \multicolumn{4}{|l|}{ Detuning energy } \\
\hline Nominal & $83.2 \mathrm{mV}(4.2 \mathrm{meV}, 1.0 \mathrm{THz})$ & & \\
\hline Error & $\begin{array}{l}0.24 \mathrm{mV}(12 \mu \mathrm{eV}, 2.8 \mathrm{GHz}) \\
\sigma=0.24 \mathrm{mV}_{\mathrm{rms}}, \mathrm{PSD}=0.24 \mu \mathrm{V} / \sqrt{\mathrm{Hz}}\end{array}$ & $167 \times 10^{-6}$ & \\
\hline \multicolumn{4}{|l|}{ Tunnel coupling } \\
\hline Nominal & $39 \mathrm{MHz}(0.16 \mu \mathrm{eV})$ & $167 \times 10^{-6}$ & $P_{\text {charge }}=99.967 \%$ \\
\hline Charge sensor & & $333 \times 10^{-6}$ & $P_{\text {sense }}=99.967 \%$ \\
\hline \multicolumn{4}{|l|}{$Q P C$} \\
\hline Signal & $400 \mathrm{pA}$ & & \\
\hline Noise & $53 \mathrm{pA}_{\mathrm{rms}}, \mathrm{PSD}=57 \mathrm{fA} / \sqrt{\mathrm{Hz}}$ & $222 \times 10^{-6}$ & \\
\hline \multicolumn{4}{|l|}{ Readout circuit } \\
\hline Input-referred noise & $26 \mathrm{pA}_{\mathrm{rms}}, \mathrm{PSD}=28 \mathrm{fA} / \sqrt{\mathrm{Hz}}$ & $111 \times 10^{-6}$ & $\begin{array}{l}P_{\text {detect }}=99.967 \% \\
F=99.9 \%\end{array}$ \\
\hline
\end{tabular}

maximum $P_{\text {charge }}$ scales with the tunnel coupling and the singlet-triplet energy splitting, as can be seen in Fig. 6(c). From this figure, an upper bound for the tunnel coupling can be found, which must be maintained even with errors caused by limitations in the control electronics.

Even though $P_{\text {charge }}$ is highly influenced by the achievable tunnel couplings and singlet-triplet energy splittings in the system [Fig. 6(c)], the detuning value has a minor influence (provided that there is a sufficient singlet-triplet energy splitting), since $1-P_{\text {charge }}$ is relatively flat around its minimum, as shown in Fig. 6(b). For instance, for a twofold increase in $1-P_{\text {charge, }}$, the detuning must stay in the range $(\epsilon-U) / E_{\mathrm{ST}} \approx 0.5 \pm 0.235$. We can then conclude that a large singlet-triplet splitting is desired to limit the influence of the control electronics on the readout.

\section{Specifications for the electronics processing the readout signal}

In this section, we will consider a direct readout. A model of a typical readout chain is shown in Fig. 6(e). For simplicity, the sensor is modeled as a current source with a value of either 0 or $I_{s}$, depending on the sensed charge. The readout fidelity is limited by the noise introduced by the sensor and by the readout circuit, indicated in Fig. 6(e) as $i_{n, s}$ and $i_{n, c}$, respectively. Assuming the typical matchedfilter detection [89], i.e., integrating the signal current for a duration $T_{\text {read }}$ and comparing the result to a threshold, the probability of a correct measurement under the presence of Gaussian-distributed noise is given by the following:

$$
P_{\text {detect }}=\frac{1+\operatorname{erf}\left(\sqrt{\frac{S / N}{8}}\right)}{2}
$$

with

$$
S / N=\frac{I_{s}^{2}}{\int_{0}^{\infty} S_{i}(f)\left[\frac{\sin \left(\pi f T_{\text {read }}\right)}{\pi f}\right]^{2} d f},
$$

where $S_{i}(f)$ is the PSD of the total noise $i_{n}=i_{n, s}+i_{n, c}$. When the noise is white, this simplifies to the following:

$$
S / N=\frac{I_{s}^{2}}{S_{i} \mathcal{B}_{\mathrm{ENBW}}}
$$

with effective noise bandwidth $\mathcal{B}_{\mathrm{ENBW}}=1 /\left(2 T_{\text {read }}\right)$.

\section{Case study of the specifications for a qubit readout}

The example specifications presented in this section build on those presented in Secs. IV C and V C and hence assume the same charging energy and lever arm for the detuning control. For the singlet-triplet energy splitting, a typical value of $E_{\mathrm{ST}}=50 \mu \mathrm{eV}$ is used. As a result, the optimum detuning is at $83.2 \mathrm{mV}$. The resulting specifications are summarized in Table $\mathrm{V}$ and assume equal contributions from $P_{\text {charge }}, P_{\text {sense }}$, and $P_{\text {detect }}$ [Eq. (15)].

Following Fig. 6(c), to achieve the required $P_{\text {charge, }}$, the tunnel coupling must be even lower than is required to turn off the two-qubit operation (see Sec. VC), thereby extending the required tunnel-coupling tuning range to approximately $18 \times$. In the example of Table $\mathrm{V}$, the detuning control can be achieved by an AWG with low sample rate, as the detuning must change adiabatically and the readout generally takes a relatively long time. Assuming that the AWG has to cover a voltage range from 0 to $2 U$, it must have a 9-bit resolution to meet the accuracy specification of the detuning energy [Fig. 6(b)]. As a result, the same circuitry as used for the two-qubit operation (Sec. V C) could potentially be used. 
In this example, $P_{\text {detect }}$ assumes a direct readout of a QPC, following the numbers provided in Ref. [64] $\left(I_{s}=\right.$ $400 \mathrm{pA}$ and $i_{n, s}=57 \mathrm{fA} / \sqrt{\mathrm{Hz}}$ ). Assuming that the readout circuit is designed to contribute about half the noise compared to the shot-noise limit of the QPC $\left(i_{n, c} \approx i_{n, s} / 2\right)$, an integration time of at least $T_{\text {read }}=0.6 \mu \mathrm{s}$ is required to achieve an signal-to-noise ratio (SNR) of 46 for a $P_{\text {detect }}$ of $99.967 \%$ [for $F=99.9 \%$, Eq. (15)]. For such short readout times, the assumption of white noise is valid and the effects of qubit relaxation $\left(T_{1}\right)$ could be negligible.

\section{DISCUSSION}

Case studies targeting a $99.9 \%$ average gate fidelity have been presented in Secs. IV C, V C, and VID. This target fidelity is particularly relevant since the minimum error rate to reach fault-tolerant quantum computing is around $99 \%$ for a complete error correction cycle, thus requiring a single-operation fidelity above $99.9 \%$ for a typical cycle of ten operations [14]. Reaching a 99.9\% fidelity for all operations is currently an ambitious goal for all qubit platforms. However, our model can be directly applied to analyze specifications for any given fidelity.

The derived electronic specifications can now be compared to the performance achieved by state-of-the-art electronics. To this end, Table VI summarizes the performance of commonly used AWGs and microwave vector sources [90-94].

For the generation of the detuning control and the microwave envelope, an AWG is required. We compare the specifications of the Tektronix 5014C, as used in, e.g., Refs. [44,52,56], with the specifications derived in the case study. This AWG achieves a sample rate of 1.2 GS/s with 14-bit resolution, thereby providing enough resolution for the amplitude and duration of the microwave envelope (150 MS/s and 8 bits). The worst-case spuriousfree dynamic range of $-56 \mathrm{dBc}$ is well below the required $-41 \mathrm{dBc}$. The specified random jitter of $5.0 \mathrm{ps}_{\mathrm{rms}}$ is well below the required value of $3.6 \mathrm{~ns}_{\mathrm{rms}}$. Finally, the output noise level is not clearly specified but can be assumed to be not much larger than the amplitude resolution of $1 \mathrm{mV}$.
In the case in which the AWG's output is attenuated by approximately $40 \mathrm{~dB}$, this also meets the specifications. This AWG can also be used for detuning control in twoqubit gates. The sample rate is high enough to meet the required timing resolution $(>1 \mathrm{GS} / \mathrm{s}$ ) and the resolution is sufficiently high to reach the detuning requirements (a worst case $0.10 \mathrm{mV}$ for a $<100 \mathrm{mV}$ pulse) with a $20 \mathrm{~dB}$ attenuator. As the specifications for Pauli-spin blockade readout are found to be more relaxed, the same AWG again suffices.

Finally, for the generation of the microwave carrier, some setups use the Agilent MW vector source E8267D $[12,44,45,52,55,56]$, which has a frequency resolution well below the requirements $(1 \mathrm{mHz}$ versus approximately 20 $\mathrm{kHz}$ ). The single-sided phase noise is also well below the required $-106 \mathrm{dBc} / \mathrm{Hz}$ at a $1 \mathrm{MHz}$ offset from the carrier (at the worst point, the E8267D achieves better than $-100 \mathrm{dBc} / \mathrm{Hz}$ at a $100 \mathrm{kHz}$ offset). The broadband noise is specified as $63 \mathrm{nV} / \sqrt{\mathrm{Hz}}(-141 \mathrm{dBc} / \mathrm{Hz}$ at $10 \mathrm{dBm})$ and therefore at least $20 \mathrm{~dB}$ attenuation is required to meet the specification of $7.1 \mathrm{nV} / \sqrt{\mathrm{Hz}}$

It can be concluded that typically adopted instruments are capable of supporting a 99.9\% fidelity. However, for the currently used instrumentation, the specifications on the amplitude noise and wide-band additive noise are the most stringent and consequently require the use of attenuators to reduce the noise reaching the quantum devices. Moreover, these instruments are bulky, consume several watts of power, and cannot be operated at cryogenic temperatures, therefore hindering scalability.

Fully integrated complementary metal-oxide semiconductor (CMOS) circuits operating at cryogenic temperatures can be adopted to tackle this problem [5,15-20]. In order to assess the feasibility of such a solution, the power consumption of the required circuit blocks will be estimated by using room-temperature CMOS circuits as a reference. This is valid, since cryogenic CMOS circuits are expected to show significantly less noise for the same power budget, as shown in Refs. [5] and [19]. As a result, the estimates given here likely overestimate the required power consumption. Furthermore, we assume a $50-\Omega$ load

TABLE VI. Specifications of commonly used AWGs and microwave vector sources.

\begin{tabular}{|c|c|c|c|c|c|}
\hline & Sample rate & Resolution & Jitter & Output noise & Spurious-free dynamic range \\
\hline Tektronix AWG5014C [90] & $1.2 \mathrm{GS} / \mathrm{s}$ & 14 bit & $5.0 \mathrm{ps}_{\mathrm{rms}}$ & $-^{\mathrm{a}}$ & $<-56 \mathrm{dBc}$ \\
\hline Keysight M9330A [91] & $1.25 \mathrm{GS} / \mathrm{s}$ & 15 bit & - & $-150 \mathrm{dBc} / \mathrm{Hz}$ & $<-65 \mathrm{dBc}$ \\
\hline \multirow[t]{2}{*}{ Tabor WX1282C [92] } & $1.25 \mathrm{GS} / \mathrm{s}$ & 14 bit & - & - & $<-44 \mathrm{dBc}$ \\
\hline & $\begin{array}{l}\text { Maximum } \\
\text { output } \\
\text { frequency }\end{array}$ & $\begin{array}{l}\text { Frequency } \\
\text { resolution }\end{array}$ & $\begin{array}{l}\text { Phase noise } \\
(100 \mathrm{kHz})\end{array}$ & $\begin{array}{l}\text { Wide-band } \\
\text { noise }(10 \mathrm{dBm})\end{array}$ & \\
\hline Agilent E8267D [93] & $44 \mathrm{GHz}$ & $1 \mathrm{mHz}$ & $<-100 \mathrm{dBc} / \mathrm{Hz}$ & $<-141 \mathrm{dBc} / \mathrm{Hz}$ & \\
\hline R\&S SMW200A [94] & $40 \mathrm{GHz}$ & $1 \mathrm{mHz}$ & $<-100 \mathrm{dBc} / \mathrm{Hz}$ & $<-134 \mathrm{dBc} / \mathrm{Hz}$ & \\
\hline
\end{tabular}

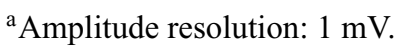


for each circuit, which is not the case for a fully integrated controller.

The core component determining the specifications of an AWG is its digital-to-analog converter (DAC). The 10-bit $500 \mathrm{MS} / \mathrm{s}$ DAC presented in Ref. [95] meets the specifications for the microwave envelope generation at a power consumption of $24 \mathrm{~mW}$. For the detuning control, the DAC specifications are stricter, but can be met by the 12-bit 1.6 GS/s DAC presented in Ref. [96], with a power consumption of $40 \mathrm{~mW}$. Although for the tunnel barrier the specifications will depend highly on the gate structure, a similar DAC is assumed to be sufficient.

The core component of a microwave carrier generator, the PLL, is also available as a CMOS circuit operating over the required frequency range $(9.2-12.7 \mathrm{GHz})$ at a power consumption of around $13 \mathrm{~mW}$ [97]. Its phase-noise performance is slightly worse than required. However, with operation at cryogenic temperatures, the noise level is expected to improve.

In a linear qubit array, one DAC is required for the barrier gate and one for the plunger gate for each qubit. This leads to an estimated power of $80 \mathrm{~mW}$ per qubit. For the microwave signals, the envelope DAC and PLL together consume approximately $40 \mathrm{~mW}$. Without any form of multiplexing, this indicates a power consumption of 120 $\mathrm{mW}$ /qubit. For a state-of-the-art dilution refrigerator with a cooling power of a few watts at $4 \mathrm{~K}$, this suggests a maximum of a few tens of qubits when operating the classical controller at $4 \mathrm{~K}$.

However, the power consumption of the DACs controlling the barrier gates and plunger gates could be highly reduced if it is not being limited to a $50-\Omega$ system. To get a sufficient signal swing, in Ref. [96] a 16-mA current is delivered to a $50-\Omega$ load, thereby setting a lower bound to the power consumption. A much lower current would be required for a higher impedance, or even for a lower swing as acceptable in this application, ultimately limited by the speed or noise requirement. Furthermore, the same fast DAC can be used to generate frequencymultiplexed microwave envelopes. With a sample rate of 1.6 GS/s, a bandwidth of roughly $640 \mathrm{MHz}$ is available [96]. This can be used to drive 64 qubits with a Rabi freqency of $1 \mathrm{MHz}$ spaced by $10 \mathrm{MHz}$ using, e.g., a Gaussian envelope [Fig. 4(d)]. The combined power of the fast DAC and PLL, i.e., $53 \mathrm{~mW}$, is then shared over 64 qubits, thus resulting in a power consumption below $1 \mathrm{~mW} /$ qubit. For the readout, on the other hand, cryogenic CMOS circuits have already been proposed that can achieve a power consumption $<1 \mathrm{~mW} /$ qubit $[5,19]$.

In summary, a cryogenic CMOS controller for a largescale quantum processor appears to be feasible for a target fidelity of $99.9 \%$. However, for minimum power consumption, the trade-offs in the electronics design must be systematically investigated. The analysis proposed in this paper provides the foundations for such optimization and will help electronics designers to build a functional controller.

\section{CONCLUSION}

In this paper, the effect of nonidealities in the classical controller for a quantum processor is analyzed. Even though this work focuses on single-electron spin-qubits, the presented approach can be used to analyze the performance of a quantum processor in any qubit technology. A comprehensive approach is proposed, by covering the effect of both static and dynamic errors on all quantum operations, i.e., single-qubit gates, two-qubit gates, and readout.

With the results of this analysis, the impact of the controller on the performance of the quantum computer as a whole can be quantified. This is required to ensure that the controller does not become the performance bottleneck as the qubit performance keeps improving. Moreover, with the presented results, a full set of electrical specifications can be derived, targeting a given qubit fidelity. The availability of these specifications enables the design of next-generation controllers that are tailor-made for the quantum processor and optimized for performance, power, cost, and size, so as to improve the scalability of the quantum computer.

As future controllers might have to operate physically close to the quantum processor, i.e., at cryogenic temperatures where the cooling power is limited, the power optimization of the controller will be critical in enabling large-scale quantum computing. With the results obtained in this paper, the trade-offs between qubit fidelity and power spent in the controller can be analyzed, this representing the foundation for such a power optimization.

\section{ACKNOWLEDGMENTS}

The authors would like to thank Intel Corp. for funding and Dr. Andrea Corna and Richard Versluis for the many interesting discussions and their useful insights.

[1] A. Montanaro, Quantum algorithms: An overview, npj Quantum Inf. 2, 15023 EP (2016).

[2] P. W. Shor, in Proceedings of 35th Annual Symposium on Foundations of Computer Science (IEEE, Santa Fe, 1994), p. 124.

[3] L. K. Grover, in Proceedings of the Twenty-Eighth annual ACM symposium on Theory of computing (ACM, New York, 1996), p. 212.

[4] R. P. Feynman, Simulating physics with computers, Int. J. Theor. Phys. 21, 467 (1982).

[5] B. Patra, R. M. Incandela, J. P. G. van Dijk, H. A. R. Homulle, L. Song, M. Shahmohammadi, R. B. Staszewski, 
A. Vladimirescu, M. Babaie, F. Sebastiano, and E. Charbon, Cryo-CMOS circuits and systems for quantum computing applications, IEEE J. Solid-State Circuits 53, 309 (2018).

[6] IBM Research, IBM builds its most powerful universal quantum computing processors (2017).

[7] J. Kelly et al., State preservation by repetitive error detection in a superconducting quantum circuit, Nature 519, 66 (2015).

[8] T. Monz, P. Schindler, J. T. Barreiro, M. Chwalla, D. Nigg, W. A. Coish, M. Harlander, W. Hänsel, M. Hennrich, and R. Blatt, 14-Qubit Entanglement: Creation and Coherence, Phys. Rev. Lett. 106, 130506 (2011).

[9] Zurich Instruments, HDAWG Arbitrary Waveform Generator (2018).

[10] Quantum Researchers Toolkit + Labber, Keysight Technologies (2017).

[11] J. P. van Dijk, E. Charbon, and F. Sebastiano, The electronic interface for quantum processors, Microprocess. Microsyst. 66, 90 (2019).

[12] J. Yoneda, K. Takeda, T. Otsuka, T. Nakajima, M. Delbecq, G. Allison, T. Honda, T. Kodera, S. Oda, Y. Hoshi, N. Usami, K. Itoh, and S. Tarucha, A > 99.9\%-fidelity quantum-dot spin qubit with coherence limited by charge noise, arXiv:1708.01454 (2017).

[13] D. Wecker, B. Bauer, B. K. Clark, M. B. Hastings, and M. Troyer, Gate-count estimates for performing quantum chemistry on small quantum computers, Phys. Rev. A 90, 022305 (2014).

[14] A. G. Fowler, M. Mariantoni, J. M. Martinis, and A. N. Cleland, Surface codes: Towards practical largescale quantum computation, Phys. Rev. A 86, 032324 (2012).

[15] J. M. Hornibrook, J. I. Colless, I. D. Conway Lamb, S. J. Pauka, H. Lu, A. C. Gossard, J. D. Watson, G. C. Gardner, S. Fallahi, M. J. Manfra, and D. J. Reilly, Cryogenic Control Architecture for Large-Scale Quantum Computing, Phys. Rev. Appl. 3, 024010 (2015).

[16] I. Conway Lamb, J. Colless, J. Hornibrook, S. Pauka, S. Waddy, M. Frechtling, and D. Reilly, An FPGA-based instrumentation platform for use at deep cryogenic temperatures, Rev. Sci. Instrum. 87, 014701 (2016).

[17] H. Homulle, S. Visser, B. Patra, G. Ferrari, E. Prati, C. G. Almudéver, K. Bertels, F. Sebastiano, and E. Charbon, in Proceedings of the ACM International Conference on Computing Frontiers, CF '16 (ACM, New York, NY, USA, 2016), p. 282.

[18] E. Charbon, F. Sebastiano, A. Vladimirescu, H. Homulle, S. Visser, L. Song, and R. M. Incandela, in 2016 IEEE International Electron Devices Meeting (IEDM) (IEEE, San Francisco, 2016), p. 13.5.1.

[19] E. Charbon, F. Sebastiano, M. Babaie, A. Vladimirescu, M. Shahmohammadi, R. B. Staszewski, H. A. R. Homulle, B. Patra, J. P. G. van Dijk, R. M. Incandela, L. Song, and B. Valizadehpasha, in 2017 IEEE International Solid-State Circuits Conference (ISSCC) (IEEE, San Francisco, 2017), p. 264.

[20] F. Sebastiano, H. Homulle, B. Patra, R. Incandela, J. van Dijk, L. Song, M. Babaie, A. Vladimirescu, and E. Charbon, in Proceedings of the 54th Annual Design Automation
Conference 2017, DAC '17 (ACM, New York, NY, USA, 2017), p. 13:1.

[21] X. Qin, Z. Shi, Y. Xie, L. Wang, X. Rong, W. Jia, W. Zhang, and J. Du, An integrated device with high performance multi-function generators and time-to-digital convertors, Rev. Sci. Instrum. 88, 014702 (2017).

[22] X. Fu, M. Rol, C. Bultink, J. van Someren, N. Khammassi, I. Ashraf, R. Vermeulen, J. De Sterke, W. Vlothuizen, R. Schouten, C. Almudever, L. DiCarlo, and K. Bertels, in Proceedings of the 50th Annual IEEE/ACM International Symposium on Microarchitecture (ACM, Cambridge, 2017), p. 813.

[23] C. A. Ryan, B. R. Johnson, D. Ristè, B. Donovan, and T. A. Ohki, Hardware for dynamic quantum computing, Rev. Sci. Instrum. 88, 104703 (2017).

[24] G. Batey, A. J. Matthews, and M. Patton, A new ultralowtemperature cryogen-free experimental platform, J. Phys. Conf. Ser. 568, 032014 (2014). IOP Publishing.

[25] L. Tavian, Latest Developments in Cryogenics at CERN, Tech. Rep. CERN-AT-2005-011-ACR (CERN, Geneva, 2005).

[26] H. Ball, W. D. Oliver, and M. J. Biercuk, The role of master clock stability in quantum information processing, npj Quantum Inf. 2, 16033 EP (2016).

[27] J. Raftery, A. Vrajitoarea, G. Zhang, Z. Leng, S. Srinivasan, and A. Houck, Direct digital synthesis of microwave waveforms for quantum computing, arXiv:1703.00942 (2017).

[28] T. J. Green, H. Uys, and M. J. Biercuk, High-Order Noise Filtering in Nontrivial Quantum Logic Gates, Phys. Rev. Lett. 109, 020501 (2012).

[29] T. J. Green, J. Sastrawan, H. Uys, and M. J. Biercuk, Arbitrary quantum control of qubits in the presence of universal noise, New J. Phys. 15, 095004 (2013).

[30] M. D. Reed, B. M. Maune, R. W. Andrews, M. G. Borselli, K. Eng, M. P. Jura, A. A. Kiselev, T. D. Ladd, S. T. Merkel, I. Milosavljevic, E. J. Pritchett, M. T. Rakher, R. S. Ross, A. E. Schmitz, A. Smith, J. A. Wright, M. F. Gyure, and A. T. Hunter, Reduced Sensitivity to Charge Noise in Semiconductor Spin Qubits via Symmetric Operation, Phys. Rev. Lett. 116, 110402 (2016).

[31] F. Martins, F. K. Malinowski, P. D. Nissen, E. Barnes, S. Fallahi, G. C. Gardner, M. J. Manfra, C. M. Marcus, and F. Kuemmeth, Noise Suppression Using Symmetric Exchange Gates in Spin Qubits, Phys. Rev. Lett. 116, 116801 (2016).

[32] N. Didier, E. A. Sete, J. Combes, and M. P. da Silva, AC flux sweet spots in parametrically-modulated superconducting qubits, arXiv:1807.01310 (2018).

[33] S. Anton, C. Müller, J. Birenbaum, S. O’Kelley, A. Fefferman, D. S. Golubev, G. Hilton, H.-M. Cho, K. Irwin, F. C. Wellstood, G. Schön, A. Shnirman, and J. Clarke, Pure dephasing in flux qubits due to flux noise with spectral density scaling as $1 / f \alpha$, Phys. Rev. B. 85, 224505 (2012).

[34] P. Rebentrost, I. Serban, T. Schulte-Herbrüggen, and F. Wilhelm, Optimal Control of a Qubit Coupled to a NonMarkovian Environment, Phys. Rev. Lett. 102, 090401 (2009).

[35] Y. V. Nazarov, Quantum Noise in Mesoscopic Physics (Springer Science \& Business Media, Delft, 2012), Vol. 97. 
[36] L. M. K. Vandersypen, M. Steffen, G. Breyta, C. S. Yannoni, R. Cleve, and I. L. Chuang, Experimental Realization of an Order-Finding Algorithm with an NMR Quantum Computer, Phys. Rev. Lett. 85, 5452 (2000).

[37] L. M. K. Vandersypen, Experimental quantum computation with nuclear spins in liquid solution, Ph.D. thesis, Stanford University, 2001.

[38] L. M. K. Vandersypen and I. L. Chuang, NMR techniques for quantum control and computation, Rev. Mod. Phys. 76, 1037 (2005).

[39] J. I. Cirac and P. Zoller, Quantum Computations with Cold Trapped Ions, Phys. Rev. Lett. 74, 4091 (1995).

[40] Y. Nakamura, Y. A. Pashkin, and J. Tsai, Coherent control of macroscopic quantum states in a single-Cooper-pair box, Nature 398, 786 (1999).

[41] Y. Makhlin, G. Schön, and A. Shnirman, Quantum-state engineering with Josephson-junction devices, Rev. Mod. Phys. 73, 357 (2001).

[42] L. Childress, M. G. Dutt, J. Taylor, A. Zibrov, F. Jelezko, J. Wrachtrup, P. Hemmer, and M. Lukin, Coherent dynamics of coupled electron and nuclear spin qubits in diamond, Science 314, 281 (2006).

[43] L. R. Schreiber and H. Bluhm, Quantum computation: Silicon comes back, Nat. Nanotechnol. 9, 966 (2014).

[44] E. Kawakami, P. Scarlino, D. R. Ward, F. R. Braakman, D. E. Savage, M. G. Lagally, M. Friesen, S. N. Coppersmith, M. A. Eriksson, and L. M. K. Vandersypen, Electrical control of a long-lived spin qubit in a $\mathrm{Si} / \mathrm{SiGe}$ quantum dot, Nat. Nanotechnol. 9, 666 EP (2014).

[45] M. Veldhorst, J. C. C. Hwang, C. H. Yang, A. W. Leenstra, B. de Ronde, J. P. Dehollain, J. T. Muhonen, F. E. Hudson, K. M. Itoh, A. Morello, and A. S. Dzurak, An addressable quantum dot qubit with fault-tolerant control-fidelity, Nat. Nanotechnol. 9, 981 EP (2014).

[46] L. M. K. Vandersypen, H. Bluhm, J. S. Clarke, A. S. Dzurak, R. Ishihara, A. Morello, D. J. Reilly, L. R. Schreiber, and $\mathrm{M}$. Veldhorst, Interfacing spin qubits in quantum dots and donors - hot, dense, and coherent, npj Quantum Inf. 3, 34 (2017).

[47] M. Veldhorst, H. G. J. Eenink, C. H. Yang, and A. S. Dzurak, Silicon CMOS architecture for a spin-based quantum computer, Nat. Commun. 8, 1766 (2017).

[48] R. Li, L. Petit, D. P. Franke, J. P. Dehollain, J. Helsen, M. Steudtner, N. K. Thomas, Z. R. Yoscovits, K. J. Singh, S. Wehner, L. Vandersypen, J. Clarke, and M. Veldhorst, A crossbar network for silicon quantum dot qubits, arXiv:1711.03807 (2017).

[49] R. Hanson, L. P. Kouwenhoven, J. R. Petta, S. Tarucha, and L. M. K. Vandersypen, Spins in few-electron quantum dots, Rev. Mod. Phys. 79, 1217 (2007).

[50] F. A. Zwanenburg, A. S. Dzurak, A. Morello, M. Y. Simmons, L. C. Hollenberg, G. Klimeck, S. Rogge, S. N. Coppersmith, and M. A. Eriksson, Silicon quantum electronics, Rev. Mod. Phys. 85, 961 (2013).

[51] J. R. Petta, A. C. Johnson, J. M. Taylor, E. A. Laird, A. Yacoby, M. D. Lukin, C. M. Marcus, M. P. Hanson, and A. C. Gossard, Coherent manipulation of coupled electron spins in semiconductor quantum dots, Science 309, 2180 (2005).

[52] E. Kawakami, T. Jullien, P. Scarlino, D. R. Ward, D. E. Savage, M. G. Lagally, V. V. Dobrovitski, M. Friesen, S.
N. Coppersmith, M. A. Eriksson, and L. M. K. Vandersypen, Gate fidelity and coherence of an electron spin in an $\mathrm{Si} / \mathrm{SiGe}$ quantum dot with micromagnet, Proc. Natl. Acad. Sci. U.S.A. 113, 11738 (2016).

[53] S. J. Angus, A. J. Ferguson, A. S. Dzurak, and R. G. Clark, Gate-defined quantum dots in intrinsic silicon, Nano Lett. 7, 2051 (2007).

[54] D. M. Zajac, T. M. Hazard, X. Mi, E. Nielsen, and J. R. Petta, Scalable Gate Architecture for a One-Dimensional Array of Semiconductor Spin Qubits, Phys. Rev. Appl. 6, 054013 (2016).

[55] M. Veldhorst, C. H. Yang, J. C. C. Hwang, W. Huang, J. P. Dehollain, J. T. Muhonen, S. Simmons, A. Laucht, F. E. Hudson, K. M. Itoh, A. Morello, and A. S. Dzurak, A two-qubit logic gate in silicon, Nature 526, 410 (2015), letter.

[56] T. F. Watson, S. G. J. Philips, E. Kawakami, D. R. Ward, P. Scarlino, M. Veldhorst, D. E. Savage, M. G. Lagally, M. Friesen, S. N. Coppersmith, M. A. Eriksson, and L. M. K. Vandersypen, A programmable two-qubit quantum processor in silicon, Nature 555, 633 (2018).

[57] The Larmor frequency can be controlled by a magnetic field gradient - e.g., by using an on-chip micromagnet [44,52,56,59] [not shown in Fig. 1(a)] or, alternatively, using the Stark shift, i.e., by an electrical field provided with an extra confinement gate $[45,55]$ [not shown in Fig. 1(a)].

[58] J. P. Dehollain, J. J. Pla, E. Siew, K. Y. Tan, A. S. Dzurak, and A. Morello, Nanoscale broadband transmission lines for spin qubit control, Nanotechnology 24, 015202 (2013).

[59] Y. Tokura, W. G. van der Wiel, T. Obata, and S. Tarucha, Coherent Single Electron Spin Control in a Slanting Zeeman Field, Phys. Rev. Lett. 96, 047202 (2006).

[60] R. Maurand, X. Jehl, D. Kotekar-Patil, A. Corna, H. Bohuslavskyi, R. Laviéville, L. Hutin, S. Barraud, M. Vinet, M. Sanquer, and S. De Fransceschi, A CMOS silicon spin qubit, Nat. Commun. 7, 13575 (2016).

[61] J. I. Colless, A. C. Mahoney, J. M. Hornibrook, A. C. Doherty, H. Lu, A. C. Gossard, and D. J. Reilly, Dispersive Readout of a Few-Electron Double Quantum Dot with Fast RF Gate Sensors, Phys. Rev. Lett. 110, 046805 (2013).

[62] A. West, B. Hensen, A. Jouan, T. Tanttu, C. Yang, A. Rossi, M. Gonzalez-Zalba, F. Hudson, A. Morello, D. Reilly, and A. Dzurak, Gate-based single-shot readout of spins in silicon, arXiv:1809.01864 (2018).

[63] D. J. Reilly, C. M. Marcus, M. P. Hanson, and A. C. Gossard, Fast single-charge sensing with a RF quantum point contact, Appl. Phys. Lett. 91, 162101 (2007).

[64] I. T. Vink, T. Nooitgedagt, R. N. Schouten, L. M. K. Vandersypen, and W. Wegscheider, Cryogenic amplifier for fast real-time detection of single-electron tunneling, Appl. Phys. Lett. 91, 123512 (2007).

[65] J. M. Elzerman, R. Hanson, L. H. W. Van Beveren, B. Witkamp, L. M. K. Vandersypen, and L. P. Kouwenhoven, Single-shot read-out of an individual electron spin in a quantum dot, Nature 430, 431 (2004).

[66] C. Barthel, M. Kjærgaard, J. Medford, M. Stopa, C. M. Marcus, M. P. Hanson, and A. C. Gossard, Fast sensing of 
double-dot charge arrangement and spin state with a radiofrequency sensor quantum dot, Phys. Rev. B 81, 161308 (2010).

[67] M. A. Nielsen, A simple formula for the average gate fidelity of a quantum dynamical operation, Phys. Lett. A 303, 249 (2002).

[68] L. H. Pedersen, N. M. Møller, and K. Mølmer, Fidelity of quantum operations, Phys. Lett. A 367, 47 (2007).

[69] See the Supplemental Material at http://link.aps.org/supp lemental/10.1103/PhysRevApplied.12.044054 for detailed derivations along with additional closed-form expressions.

[70] N. Khaneja, T. Reiss, C. Kehlet, T. Schulte-Herbrüggen, and S. J. Glaser, Optimal control of coupled spin dynamics: Design of NMR pulse sequences by gradient ascent algorithms, J. Magn. Reson. 172, 296 (2005).

[71] While it might be possible to calibrate for drift, this significantly increases the complexity of the system.

[72] Assuming that the rotating-wave approximation (RWA) needs to be accurate. The RWA is discussed in the Supplemental Material [69].

[73] The indicated ENBW is defined such that white noise integrated over this bandwidth results in the same noise power as white noise integrated over the actual filter transfer function and can be used to simplify calculations when the noise can be well approximated as white noise in the band of interest [75] (see the Supplemental Material [69]).

[74] Note that some quantum algorithms, such as dynamicaldecoupling sequences or error-correction codes, can act as a high-pass filter for the noise, thereby setting $\omega_{\min }$.

[75] D. A. Johns and K. Martin, Analog Integrated Circuit Design (John Wiley \& Sons, 2008).

[76] G. S. Uhrig, Keeping a Quantum Bit Alive by Optimized $\pi$-Pulse Sequences, Phys. Rev. Lett. 98, 100504 (2007).

[77] M. J. Biercuk, H. Uys, A. P. VanDevender, N. Shiga, W. M. Itano, and J. J. Bollinger, Optimized dynamical decoupling in a model quantum memory, Nature 458, 996 (2009).

[78] Solutions for Reducing Phase Noise at RF and Microwave Frequencies, Keysight Technologies (2014).

[79] J. Phillips and K. Kundert, in Proceedings of the IEEE 2000 Custom Integrated Circuits Conference (IEEE, Orlando, 2000), p. 431.

[80] B. Drakhlis, Calculate oscillator jitter by using phase-noise analysis, MICROWAVES \& RF 40, 82 (2001).

[81] M. McCoy and L. Mueller, Selective decoupling, J. Magn. Reson., Ser. A 101, 122 (1993).
[82] E. Kupce and R. Freeman, Close encounters between soft pulses, J. Magn. Reson., Ser. A 112, 261 (1995).

[83] J. Pauly, P. L. Roux, D. Nishimura, and A. Macovski, Parameter relations for the Shinnar-Le Roux selective excitation pulse design algorithm [NMR imaging], IEEE Trans. Med. Imaging 10, 53 (1991).

[84] M. Steffen, L. M. K. Vandersypen, and I. L. Chuang, Simultaneous soft pulses applied at nearby frequencies, J. Magn. Reson. 146, 369 (2000).

[85] W. A. Coish and D. Loss, Exchange-controlled singleelectron-spin rotations in quantum dots, Phys. Rev. B 75, 161302 (2007).

[86] T. Meunier, V. E. Calado, and L. M. K. Vandersypen, Efficient controlled-phase gate for single-spin qubits in quantum dots, Phys. Rev. B 83, 121403 (2011).

[87] E. A. Laird, J. R. Petta, A. C. Johnson, C. M. Marcus, A. Yacoby, M. P. Hanson, and A. C. Gossard, Effect of Exchange Interaction on Spin Dephasing in a Double Quantum Dot, Phys. Rev. Lett. 97, 056801 (2006).

[88] K. Ono, D. Austing, Y. Tokura, and S. Tarucha, Current rectification by Pauli exclusion in a weakly coupled double quantum dot system, Science 297, 1313 (2002).

[89] G. Turin, An introduction to matched filters, IRE Trans. Inf. Theory 6, 311 (1960).

[90] Arbitrary Waveform Generators AWG5000 Series Data Sheet, Tektronix (2017).

[91] Keysight M9330A Arbitrary Waveform Generator 15-bit, $1.25 \mathrm{GS} / \mathrm{s}$, Keysight Technologies (2014).

[92] MODELS WX1281/2C 1.25 GS/s Single/Dual Channel Arbitrary Waveform Generators, Tabor Electronics Inc. (2015).

[93] E8267D PSG Vector Signal Generator, Keysight Technologies (2016).

[94] R\&S SMW200A Vector Signal Generator Specifications, Rohde \& Schwarz (2018).

[95] W. T. Lin and T. H. Kuo, A compact dynamicperformance-improved current-steering DAC with random rotation-based binary-weighted selection, IEEE J. Solid-State Circuits 47, 444 (2012).

[96] W. T. Lin, H. Y. Huang, and T. H. Kuo, A 12-bit $40 \mathrm{~nm}$ DAC achieving SFDR $>70 \mathrm{~dB}$ at $1.6 \mathrm{GS} / \mathrm{s}$ and IMD $<-61 \mathrm{~dB}$ at $2.8 \mathrm{GS} / \mathrm{s}$ with DEMDRZ technique, IEEE J. Solid-State Circuits 49, 708 (2014).

[97] K. Raczkowski, N. Markulic, B. Hershberg, and J. Craninckx, A 9.2-12.7 GHz wideband fractional-N subsampling PLL in $28 \mathrm{~nm}$ CMOS with 280 fs RMS jitter, IEEE J. Solid-State Circuits 50, 1203 (2015). 